\title{
E-optimality conditions and Wolfe E-duality for E- differentiable vector optimization problems with inequality and equality constraints
}

\author{
Tadeusz Antczak ${ }^{\mathrm{a}}$, Najeeb Abdulaleem ${ }^{\mathrm{b}, *}$ \\ ${ }^{a}$ Faculty of Mathematics and Computer Science, University of Łódź, Banacha 22, 90-238 Łódź, Poland. \\ ${ }^{b}$ Department of Mathematics, Hadhramout University, P. O. BOX: (50511-50512), Al-Mahrah, Yemen.
}

\begin{abstract}
In this paper, a nonconvex vector optimization problem with both inequality and equality constraints is considered. The functions constituting it are not necessarily differentiable, but they are E-differentiable. The so-called E-Fritz John necessary optimality conditions and the so-called E-Karush-Kuhn-Tucker necessary optimality conditions are established for the considered E-differentiable multiobjective programming problems with both inequality and equality constraints. Further, the sufficient optimality conditions are derived for such nonconvex nonsmooth vector optimization problems under (generalized) E-convexity. The so-called vector E-Wolfe dual problem is defined for the considered E-differentiable multiobjective programming problem with both inequality and equality constraints and several dual theorems are established also under (generalized) E-convexity hypotheses.
\end{abstract}

Keywords: E-differentiable function, E-Fritz John necessary optimality conditions, E-Karush-Kuhn-Tucker necessary optimality conditions, E-Wolfe duality, E-convex function.

2010 MSC: 90C26, 90C29, 90C30, 90C46, 90C 47.

(C)2019 All rights reserved.

\section{Introduction}

During the last few years, multiobjective optimization (also referred to as multiobjective programming, vector optimization, and multicriteria optimization) has received great interest from the authors in the areas of optimization theory. This is a consequence of the fact that multiobjective optimization is applied in many mathematical disciplines and it is known as a useful mathematical model in order to investigate many real world problems with conflicting objectives, arising from human decision making, engineering, mechanics, economics, logistics, optimization and information technology. Necessary optimality conditions for various classes of nonlinear differentiable and nondifferentiable multiobjective programming problems have been studied extensively in the literature (see, for example, $[7,14,17,9,10,19,21,25,27,33,29]$, and others). The theory and applications of vector optimization problems have been closely tied with convex analysis. In recent years, however, there has been an increasing

\footnotetext{
${ }^{*}$ Corresponding author

Email addresses: tadeusz.antczak@wmii.uni.lodz.pl (Tadeusz Antczak), nabbas985@gmail.com (Najeeb Abdulaleem )
}

doi: 10.22436/jnsa.012.11.06 
interest in developing sufficient optimality conditions and duality results for various classes of nonconvex vector optimization problems (see, for instance, $[1,2,3,4,5,6,8,12,18,9,23,24,30,32,34,22,43]$, and others).

Classes of nonconvex sets and nonconvex functions, called E-convex sets and E-convex functions, respectively, were introduced and studied by Youness [40]. This kind of generalized convexity is based on the effect of an operator $E: R^{n} \rightarrow R^{n}$ on the sets and the domains of functions. However, some results and proofs presented by Youness [40] were incorrect as it was pointed out by Yang [39]. Further, Chen [11] introduced a new class of semi-E-convex functions and discussed its basic properties. Youness and Emam [44] derived new concepts of a strongly E-convex set and a strongly E-convex function and presented some properties of these classes of nonconvex sets and functions. Soleimani-damaneh [37] established some properties of E-convex and generalized E-convex functions. Megahed et al. [30] introduced a combined interactive approach for solving generalized E-convex multiobjective nonlinear problems. Megahed et al. [29] presented the concept of an E-differentiable convex function which transforms a (not necessarily) differentiable convex function to a differentiable function based on the effect of an operator $E: R^{n} \rightarrow R^{n}$.

In this paper, a nonconvex vector optimization problem with both inequality and equality constraints is considered. The functions constituting it are not necessarily differentiable, but they are E-differentiable. The so-called E-Fritz John necessary optimality conditions are established for the considered E-differentiable vector optimization problem. Further, under the introduced E-Abadie constraint qualification, the E-Karush-Kuhn-Tucker necessary optimality conditions are also proved for such not necessarily differentiable vector optimization problems, in which all involved functions are E-differentiable. It is also given an example of such a vector optimization problems with E-differentiable functions for which the E-Abadie constraint qualification does not hold. It turns out that the E-Karush-Kuhn-Tucker necessary optimality conditions established for such a nonsmooth vector optimization problem are not satisfied in this case. Furthermore, the sufficient optimality conditions are derived for the considered E-differentiable vector optimization problem under (generalized) E-convexity.

Furthermore, for the considered E-differentiable vector optimization problem with both inequality and equality constraints, its vector dual problem in the sense of Wolfe, called Wolfe vector E-dual problem, is defined. Then various duality theorems between the considered E-differentiable vector optimization problem and its Wolfe vector E-dual problem are established also under (generalized) E-convexity hypotheses.

\section{Preliminaries}

Throughout this paper the following conventions vectors $x=\left(x_{1}, x_{2}, \cdots, x_{n}\right)^{\top}$ and $y=\left(y_{1}, y_{2}, \cdots, y_{n}\right)^{\top}$ in $\mathrm{R}^{\mathrm{n}}$ will be followed:

(i) $x=y$ if and only if $x_{i}=y_{i}$ for all $i=1,2, \cdots, n$;

(ii) $x>y$ if and only if $x_{i}>y_{i}$ for all $i=1,2, \cdots, n$;

(iii) $x \geqq y$ if and only if $x_{i} \geqq y_{i}$ for all $i=1,2, \cdots, n$;

(iv) $x \geqslant y$ if and only if $x_{i} \geqq y_{i}$ for all $i=1,2, \cdots, n$ but $x \neq y$;

(v) $x \ngtr y$ is the negation of $x>y$.

The definition of an E-convex set and the definition of an E-convex function were introduced by Youness [40]. Now, for convenience, we recall these definitions.

Definition 2.1 ([40]). A set $M \subseteq R^{n}$ is said to be an E-convex set (with respect to an operator $E: R^{n} \rightarrow R^{n}$ ) if and only if, the following relation

$$
E(u)+\lambda(E(x)-E(u)) \in M
$$

holds for all $x, u \in M$ and any $\lambda \in[0,1]$. 
Note that every convex set is $E$-convex (if $E$ is the identity map), but the converse is not true. If $M \subseteq R^{n}$ is an $E$-convex set, then $E(M) \subseteq M$. If $E(M)$ is a convex set and $E(M) \subseteq M$, then $M$ is $E$-convex (see, Youness [42]).

Let $M$ be a nonempty E-convex subset of $R^{n}$.

Definition 2.2 ([40]). A real-valued function $f: M \rightarrow R$ is said to be E-convex (with respect to an operator $E: R^{n} \rightarrow R^{n}$ ) on $M$ if and only if, the following inequality

$$
f(\lambda E(x)+(1-\lambda) E(u)) \leqq \lambda f(E(x))+(1-\lambda) f(E(u))
$$

holds for all $x, u \in M$ and any $\lambda \in[0,1]$.

It is clear that every convex function is E-convex (if $E$ is the identity map).

Definition 2.3. A real-valued function $f: M \rightarrow R$ is said to be strictly E-convex (with respect to an operator $E: R^{n} \rightarrow R^{n}$ ) on $M$ if and only if, the following inequality

$$
f(\lambda E(x)+(1-\lambda) E(u))<\lambda f(E(x))+(1-\lambda) f(E(u))
$$

holds for all $x, u \in M, E(x) \neq E(u)$, and any $\lambda \in(0,1)$.

Definition 2.4 ([42]). A function $f: M \rightarrow R$ is said to be quasi-E-convex (with respect to an operator $E: R^{n} \rightarrow R^{n}$ ) on $M$ if and only if, the following inequality

$$
f(\lambda E(x)+(1-\lambda) E(u)) \leqq \max \{(f \circ E)(x),(f \circ E)(u)\}
$$

holds for all $x, u \in M$ and any $\lambda \in[0,1]$.

Note that every E-convex function is quasi-E-convex and every convex function is quasi-E-convex (if $E$ is the identity map).

Example 2.5. Let $f: R \rightarrow R$ be defined by $f(x)=\left\{\begin{array}{cc}1 & \text { if } x=0, \\ 0 & \text { otherwise, }\end{array}\right.$ and an operator $E: R \rightarrow R$ be defined by $E(x)=\left\{\begin{array}{lc}0 & \text { if } x=0, \\ 3 & \text { otherwise }\end{array}\right.$. Note that $f$ is neither convex nor quasi-convex. Indeed, if we set $x=1, u=-1$, and $\lambda=\frac{1}{2}$, then we have

$$
f(\lambda x+(1-\lambda) u)=1>\lambda f(x)+(1-\lambda) f(u)=\max \{f(x), f(u)\}=0 .
$$

But $f$ is both E-convex and quasi-E-convex. In order to prove this result, let $x, u \in R$ and $\lambda \in(0,1)$. Then, we consider the following cases:

(a) If $x=u=0$, then $f(\lambda E(x)+(1-\lambda) E(u))=\lambda f(E(x))+(1-\lambda) f(E(u))=1$.

(b) If $x \neq 0$ and $u \neq 0$, then $f(\lambda E(x)+(1-\lambda) E(u))=\lambda f(E(x))+(1-\lambda) f(E(u))=0$.

(c) If $x=0$ and $u \neq 0$, then $f(\lambda E(x)+(1-\lambda) E(u))=0<\lambda f(E(x))+(1-\lambda) f(E(u))=\lambda$.

(d) If $x \neq 0$ and $u=0$, then $f(\lambda E(x)+(1-\lambda) E(u))=0<\lambda f(E(x))+(1-\lambda) f(E(u))=1-\lambda$.

Therefore, we have that, in all considered cases, the following inequalities

$$
f(\lambda E(x)+(1-\lambda) E(u)) \leqq \lambda f(E(x))+(1-\lambda) f(E(u)) \leqq \max \{f(E(x)), f(E(u))\}
$$

hold for all $x, u \in R$ and any $\lambda \in[0,1]$. Hence, by Definitions 2.2 and 2.4, the function $f$ is both E-convex and quasi-E-convex. 
Definition 2.6 ([30]). Let $E: R^{n} \rightarrow R^{n}, M$ be an open E-convex set, $f: M \rightarrow R$ be a (not necessarily) differentiable function at a given point $u$. It is said that $f$ is an $E$-differentiable function at $u$ if and only if, $f \circ E$ is a differentiable function at $u$ (in the usual sense) and, moreover,

$$
(f \circ E)(x)=(f \circ E)(u)+\nabla(f \circ E)(u)(x-u)+\theta(u, x-u)\|x-u\|,
$$

where $\theta(u, x-u) \rightarrow 0$ as $x \rightarrow u$.

If $f$ is $E$-differentiable at $\bar{x}$, then there exists only one gradient vector of $f \circ E$ at $\bar{x}$ and it is given by

$$
\nabla(f \circ E)(\bar{x})=\left(\frac{\partial(f \circ E)(\bar{x})}{\partial x_{1}}, \cdots, \frac{\partial(f \circ E)(\bar{x})}{\partial x_{n}}\right) .
$$

Proposition 2.7. Let $\mathrm{E}: \mathrm{R}^{\mathrm{n}} \rightarrow \mathrm{R}^{\mathrm{n}}, \mathrm{M}$ be an $\mathrm{E}$-convex subset of $\mathrm{R}^{\mathrm{n}}, \mathrm{f}: \mathrm{M} \rightarrow \mathrm{R}$ be an $\mathrm{E}$-convex (strictly $\mathrm{E}$-convex) function on $\mathrm{M}$ and $\mathrm{u} \in \mathrm{M}$. Further, assume that $\mathrm{f}$ is $\mathrm{E}$-differentiable at $\mathrm{u}$. Then, the following inequality

$$
f(E(x))-f(E(u)) \geqq \nabla f(E(u))(E(x)-E(u)), \quad(>)
$$

holds for all $x \in M$.

Proof. Assume that $M$ is an E-convex set, $f: M \rightarrow R$ is an E-convex function on $M$ and $u \in M$. By Definition 2.2, it follows that the inequality

$$
f(\lambda E(x)+(1-\lambda) E(u)) \leqq \lambda f(E(x))+(1-\lambda) f(E(u))
$$

holds for all $x, u \in M$ and any $\lambda \in[0,1]$. Thus, the above inequality yields,

$$
f(E(x))-f(E(u)) \geqq \frac{f(E(u)+\lambda(E(x)-E(u)))-f(E(u))}{\lambda} .
$$

Letting $\lambda \rightarrow 0$, we obtain the inequality (2.1).

Now, we give the necessary condition for an E-differentiable function.

Proposition 2.8. Let $\mathrm{E}: \mathrm{R}^{\mathrm{n}} \rightarrow \mathrm{R}^{\mathrm{n}}, \mathrm{M}$ be an $\mathrm{E}$-convex subset of $\mathrm{R}^{\mathrm{n}}, \mathrm{f}: \mathrm{M} \rightarrow \mathrm{R}$ be an $\mathrm{E}$-convex (strictly $\mathrm{E}$-convex) function on $M$ and $x, u \in M$. Further, assume that $f$ is $E$-differentiable on an open set $M$. Then, the following inequality

$$
\nabla f(E(x))-\nabla f(E(u))(E(x)-E(u)) \geqq 0 \quad(>)
$$

holds for all $x, u \in M$.

Now, we give the definitions of E-differentiable generalized E-convex functions.

Definition 2.9. Let $E: R^{n} \rightarrow R^{n}, M$ be a nonempty E-convex subset of $R^{n}$ and $f: M \rightarrow R$ be an Edifferentiable function at $u \in M$. $f$ is said to be a pseudo E-convex function at $u$ on $M$ if the following relation

$$
(f \circ E)(x)<(f \circ E)(u) \Longrightarrow \nabla(f \circ E)(u)(E(x)-E(u))<0
$$

holds for all $x \in M$. If (2.2) is satisfied for each $u \in M$, then $f$ is said to be a pseudo E-convex function on M.

Definition 2.10. Let $E: R^{n} \rightarrow R^{n}, M$ be a nonempty E-convex subset of $R^{n}$ and $f: M \rightarrow R$ be an $E$ differentiable function at $u \in M$. $f$ is said to be a strictly pseudo E-convex function at $u$ on $M$ if the following relation

$$
(f \circ E)(x) \leqq(f \circ E)(u) \Longrightarrow \nabla(f \circ E)(u)(E(x)-E(u))<0
$$

holds for all $x, u \in M, E(x) \neq E(u)$. If (2.3) is satisfied for each $u \in M, x \neq u$, then $f$ is said to be a strictly pseudo $\mathrm{E}$-convex function on $\mathrm{M}$. 
Note that every E-differentiable E-convex function is pseudo E-convex, but the converse is not true. Also, every strictly pseudo E-convex function is pseudo E-convex and every differentiable convex function is pseudo E-convex (if $E$ is identity map). Every E-differentiable strictly E-convex function is strictly pseudo E-convex, but the converse is not true.

Now, we present an example of such an E-differentiable pseudo E-convex function which is not Econvex.

Example 2.11. Let $f:\left[0, \frac{\pi}{2}\right] \rightarrow R$ be defined by $f(x)=\sin x$ and $E:\left[0, \frac{\pi}{2}\right] \rightarrow\left[0, \frac{\pi}{2}\right]$ be an operator defined by $E(x)=\frac{\pi}{2}-x$. Further, assume that $(f \circ E)(x)<(f \circ E)(u)$. Thus, we have

$$
(f \circ E)(x)=\cos x<\cos u=(f \circ E)(u) .
$$

This implies that $x<u$ for all $x, u \in\left[0, \frac{\pi}{2}\right]$. Hence, we have

$$
\nabla(f \circ E)(u)(E(x)-E(u))=-\sin u(u-x)<0 .
$$

Therefore, by Definition 2.9, $\mathrm{f}$ is E-differentiable pseudo E-convex on $\left[0, \frac{\pi}{2}\right]$. Considering $x=0, u=\frac{\pi}{2}$, and $\lambda=\frac{1}{2}$, we have

$$
f(\lambda E(x)+(1-\lambda) E(u))=\frac{1}{\sqrt{2}}>\lambda f(E(x))+(1-\lambda) f(E(u))=\frac{1}{2} .
$$

Hence, by Definition 2.2, it follows that $f$ is not E-convex on $\left[0, \frac{\pi}{2}\right]$.

Definition 2.12. Let $E: R^{n} \rightarrow R^{n}, M$ be a nonempty E-convex subset of $R^{n}$ and $f: M \rightarrow R$ be an E-differentiable function at $u \in M$. $f$ is said to be quasi-E-convex function at $u$ on $M$ if the following relation

$$
(f \circ E)(x) \leqq(f \circ E)(u) \Longrightarrow \nabla(f \circ E)(u)(E(x)-E(u)) \leqq 0
$$

holds for all $x \in M$. If (2.4) is satisfied for each $u \in M$, then $f$ is said to be a quasi E-convex function on M.

Now, we present an example of a nondifferentiable function, which is both E-differentiable pseudo E-convex and quasi E-convex on R.

Example 2.13. Let $f: R \rightarrow R$ be a nondifferentiable function at $x=-2$ defined by $f(x)=(x+2)^{\frac{1}{3}}$ and $E: R \rightarrow R$ be an operator such that $E(x)=(x+2)^{9}-2$. The function $(f \circ E)(x)=(x+2)^{3}$ is a differentiable function at $x=-2$, thus $f$ is an E-differentiable function at $x=-2$.

Now we show that $f$ is pseudo E-convex on $R$. Let $x, u \in R$ and $\lambda \in[0,1]$, and assume that

$$
(f \circ E)(x)<(f \circ E)(u) \text {. }
$$

We have

$$
(f \circ E)(x)=(x+2)^{3}<(u+2)^{3}=(f \circ E)(u) .
$$

This inequality implies that $x<u$. Hence, we have

$$
\nabla(f \circ E)(u)(E(x)-E(u))=3(u+2)^{2}\left((x+2)^{9}-(u+2)^{9}\right)<0 .
$$

Therefore, by Definition 2.9, $\mathrm{f}$ is pseudo E-convex on $\mathrm{R}$.

Further, it can be shown that $f$ is also quasi E-convex on R. Assume that $(f \circ E)(x) \leqq(f \circ E)(u)$. We have $(f \circ E)(x)=(x+2)^{3} \leqq(u+2)^{3}=(f \circ E)(u)$. This inequality implies that $x \leqq u$. Hence, we have

$$
\nabla(f \circ E)(u)(E(x)-E(u))=3(u+2)^{2}\left((x+2)^{9}-(u+2)^{9}\right) \leqq 0 .
$$

Therefore, by Definition 2.12, $\mathrm{f}$ is quasi E-convex on R. 


\section{E-differentiable multiobjective programming}

Consider the following (not necessarily differentiable) multiobjective programming problem (VP) with both inequality and equality constraints:

$$
\begin{array}{r}
\text { minimize } f(x)=\left(f_{1}(x), \cdots, f_{p}(x)\right) \\
\text { subject to } g_{j}(x) \leqq 0, \quad j \in J=\{1, \cdots, m\}, \\
h_{t}(x)=0, t \in T=\{1, \cdots, q\},
\end{array}
$$

where $f_{i}: R^{n} \rightarrow R, i \in I=\{1, \cdots, p\}, g_{j}: R^{n} \rightarrow R, j \in J, h_{t}: R^{n} \rightarrow R, t \in T$, are real-valued functions defined on $R^{n}$. We shall write $g:=\left(g_{1}, \cdots, g_{m}\right): R^{n} \rightarrow R^{m}$ and $h:=\left(h_{1}, \cdots, h_{q}\right): R^{n} \rightarrow R^{q}$ for convenience.

For the purpose of simplifying our presentation, we will next introduce some notation which will be used frequently throughout this paper. Let

$$
\Omega:=\left\{x \in R^{n}: g_{j}(x) \leqq 0, j \in J, h_{t}(x)=0, t \in T\right\}
$$

be the set of all feasible solutions of (VP) (3.1). Further, by $J(x)$, we denote the set of inequality constraint indices that are active at a feasible solution $x$, that is, $J(x)=\left\{j \in J: g_{j}(x)=0\right\}$.

For such multicriterion optimization problems, the following concepts of (weak) Pareto optimal solutions are defined as follows:

Definition 3.1. A feasible point $\bar{x}$ is said to be a weak Pareto (weakly efficient) solution for (VP) (3.1) if and only if there is no another feasible solution $x$ such that

$$
f(x)<f(\bar{x}) .
$$

Definition 3.2. A feasible point $\bar{x}$ is said to be a Pareto (efficient) solution for (VP) (3.1) if and only if there is no another feasible solution $x$ such that

$$
f(x) \leqslant f(\bar{x})
$$

Let $E: R^{n} \rightarrow R^{n}$ be a given one-to-one and onto operator. Throughout the paper, we shall assume that the functions constituting the considered multiobjective programming problem (VP) (3.1) are Edifferentiable at any feasible solution.

Now, for the considered multiobjective programming problem (VP) (3.1), we define its associated differentiable vector optimization problem as follows:

$$
\begin{array}{r}
\operatorname{minimize}(f \circ E)(x)=\left(\left(f_{1} \circ E\right)(x), \cdots,\left(f_{p} \circ E\right)(x)\right) \\
\text { subject to }\left(g_{j} \circ E\right)(x) \leqq 0, j \in J=\{1, \cdots, m\}, \\
\left(h_{t} \circ E\right)(x)=0, t \in T=\{1, \cdots, q\} .
\end{array}
$$

We call the problem $\left(\mathrm{VP}_{\mathrm{E}}\right)$ (3.2) an E-vector optimization problem. Let

$$
\Omega_{E}:=\left\{x \in R^{n}:\left(g_{j} \circ E\right)(x) \leqq 0, j \in J,\left(h_{t} \circ E\right)(x)=0, t \in T\right\}
$$

be the set of all feasible solutions of $\left(\mathrm{VP}_{\mathrm{E}}\right)$ (3.2). Since the functions constituting the problem (VP) (3.1) are assumed to be E-differentiable at any feasible solution of (VP) (3.1), by Definition 2.6, the functions constituting the E-vector optimization problem $\left(\mathrm{VP}_{\mathrm{E}}\right)$ (3.2) are differentiable at any its feasible solution (in the usual sense). Further, by $\mathrm{J}_{\mathrm{E}}(\mathrm{x})$, the set of inequality constraint indices that are active at a feasible solution $x$, that is, $J_{E}(x)=\left\{j \in J:\left(g_{j} \circ E\right)(x)=0\right\}$.

Before proving the optimality conditions for (weakly) efficiency of the problem (VPE) (3.2) and, thus, (weakly) efficiency for the problem (VP) (3.1), we establish some useful results. 
Lemma 3.3. Let $\mathrm{E}: \mathrm{R}^{\mathrm{n}} \rightarrow \mathrm{R}^{\mathrm{n}}$ be a one-to-one and onto and

$$
\Omega_{E}=\left\{z \in R^{n}:\left(g_{j} \circ E\right)(z) \leqq 0, j \in J,\left(h_{t} \circ E\right)(z)=0, t \in T\right\} .
$$

Then $\mathrm{E}\left(\Omega_{\mathrm{E}}\right)=\Omega$.

Proof. Let $x \in E\left(\Omega_{E}\right)$. By assumption, $E$ is an one-to-one and onto operator. Then, by the definition of $\Omega_{E}$, there exists $z \in \Omega_{E}$ such that $x=E(z)$. We proceed by contradiction. Suppose that $x \notin \Omega$. Then there exists at least one $j \in J$ such that $g_{j}(x)>0$ or at least one $t \in T$ such that $h_{t}(x) \neq 0$. Hence, by $x=E(z)$, we have for at least one $j \in J$ that $\left(g_{j} \circ E\right)(z)>0$ or for at least one $t \in T$ that $\left(h_{t} \circ E\right)(z) \neq 0$, which contradicting $z \in \Omega_{\mathrm{E}}$. Thus, $\mathrm{E}\left(\Omega_{\mathrm{E}}\right) \subset \Omega$.

On the other hand, let $x \in \Omega$. We proceed by contradiction. Suppose that $x \notin E\left(\Omega_{E}\right)$. By assumption, this means that $E^{-1}(x) \notin \Omega_{E}$. By the definition of $\Omega_{E}$, it follows that there exists at least one $j \in J$ such that $\left(g_{j} \circ E\right)\left(E^{-1}(x)\right)>0$ or at least one $t \in T$ such that $\left(h_{t} \circ E\right)\left(E^{-1}(x)\right) \neq 0$. This means that there exists at least one $j \in J$ such that $g_{j}(x)>0$ or at least one $t \in T$ such that $h_{t}(x) \neq 0$, contradicting $x \in \Omega$. Thus, $\Omega \subset \mathrm{E}\left(\Omega_{\mathrm{E}}\right)$. By $\mathrm{E}\left(\Omega_{\mathrm{E}}\right) \subset \Omega$ and $\Omega \subset \mathrm{E}\left(\Omega_{\mathrm{E}}\right)$, we conclude that $\mathrm{E}\left(\Omega_{\mathrm{E}}\right)=\Omega$.

Now, we prove the relationship between (weak) Pareto optimal solutions in both vector optimization problems (VP) (3.1) and ( $\left.\mathrm{VP}_{\mathrm{E}}\right)$ (3.2).

Lemma 3.4. Let $\bar{x} \in \Omega$ be a weak Pareto solution (Pareto solution) of the considered multiobjective programming problem (VP) (3.1). Then, there exists $\bar{z} \in \Omega_{\mathrm{E}}$ such that $\overline{\mathrm{x}}=\mathrm{E}(\bar{z})$ and $\bar{z}$ is a weak Pareto (Pareto) solution of the E-vector optimization problem $\left(V P_{\mathrm{E}}\right)(3.2)$.

Proof. Let $\bar{x} \in \Omega$ be a weak Pareto solution for the considered $E$-differentiable multiobjective programming problem (VP) (3.1). By Lemma 3.3, it follows that there exists $\bar{z} \in \Omega_{E}$ such that $\bar{x}=\mathrm{E}(\bar{z})$. Now, we prove that $\bar{z}$ is a weak Pareto solution of the E-vector optimization problem (VPE) (3.2). By means of contradiction, suppose that $\bar{z}$ is not a weak Pareto solution of the E-vector optimization problem $\left(\mathrm{VP}_{\mathrm{E}}\right)$ (3.2). Then, by Definition 3.1, there exists $\widetilde{z} \in \Omega_{E}$ such that $(f \circ E)(\widetilde{z})<(f \circ E)(\bar{z})$. By Lemma 3.3, we have that there exists $\widetilde{x} \in \Omega$ such that $\widetilde{x}=E(\widetilde{z})$. Hence, the inequality above implies that $f(\widetilde{x})<f(\bar{x})$, which is a contradiction to weakly efficiency of $\bar{x}$ for the problem (VP) (3.1). The proof in the case when $\bar{x} \in \Omega$ is a Pareto solution of the problem (VP) (3.1) is similar.

Lemma 3.5. Let $\bar{z} \in \Omega_{\mathrm{E}}$ be a weak Pareto (Pareto) solution of the E-vector optimization problem (VP $\mathrm{E}$ ) (3.2). Then $\mathrm{E}(\bar{z})$ is a weak Pareto solution (Pareto solution) of the considered multiobjective programming problem (VP) (3.1).

Proof. Assume that $\bar{z} \in \Omega_{\mathrm{E}}$ is a weak Pareto solution of the E-vector optimization problem (VP $\mathrm{E}_{\mathrm{E}}$ ) (3.2). Note that, by Lemma 3.3, it follows that $E(\bar{z}) \in \Omega$. We proceed by contradiction. Suppose, contrary to the result, that $E(\bar{z})$ is not a weak Pareto solution for the considered multiobjective programming problem (VP) (3.1). Then, by Definition 3.1, there exists $\widetilde{x} \in \Omega$ such that $f(\widetilde{x})<f(E(\bar{z}))$. Using Lemma 3.3, there exists $\widetilde{z} \in \Omega_{E}$ such that $\widetilde{x}=E(\widetilde{z})$. Thus, the inequality above implies that $(f \circ E)(\widetilde{z})<(f \circ E)(\bar{z})$, which is a contradiction to weakly efficiency of $\bar{z}$ for the problem $\left(\mathrm{VP}_{\mathrm{E}}\right)$ (3.2). The proof in the case when $\bar{z} \in \Omega_{\mathrm{E}}$ is a Pareto solution of the problem $\left(\mathrm{VP}_{\mathrm{E}}\right)(3.2)$ is similar.

Remark 3.6. As it follows from Lemma 3.5, if $\bar{z} \in \Omega_{\mathrm{E}}$ is a weak Pareto (Pareto) solution of the E-vector optimization problem $\left(\mathrm{VP}_{\mathrm{E}}\right)$ (3.2), then $\mathrm{E}(\bar{z})$ is a weak Pareto solution (Pareto solution) of the considered multiobjective programming problem (VP) (3.1). We call $E(\bar{z})$ a weak E-Pareto (E-Pareto) solution of the problem (VP) (3.1).

As it follows from the above lemmas, there is some equivalence between the vector optimization problem (VP) (3.1) and ( $\left.\mathrm{VP}_{\mathrm{E}}\right)$ (3.2). Therefore, if we prove optimality results for the differentiable $\mathrm{E}$ vector optimization problem $\left(\mathrm{VP}_{\mathrm{E}}\right)$ (3.2), they will be applicable also for the original nondifferentiable multiobjective programming problem (VP) (3.1), in which the involved functions are E-differentiable. 
Now, we prove the Fritz John necessary optimality conditions for $\bar{x} \in \Omega_{\mathrm{E}}$ to be a weak Pareto solution of the differentiable E-vector optimization problem which are also the so-called E-Fritz John necessary optimality conditions for $E(\bar{x})$ to be a weak E-Pareto solution of the considered multiobjective programming problem (VP) (3.1). Before we establish them, we recall the Motzkin's theorem of the alternative which we use in proving this result.

Theorem 3.7 (Motzkin's theorem of the alternative [28]). Let A, C, D be given matrices, with A being nonvacuous. Then either the system

$$
\mathrm{A} x<0, \mathrm{C} x \leqq 0, \mathrm{D} x=0
$$

has a solution $x$, or the system

$$
A^{\top} y_{1}+C^{\top} y_{2}+D^{\top} y_{3}=0, \quad y_{1} \geqslant 0, y_{2} \geqq 0
$$

has solution $\mathrm{y}_{1}, \mathrm{y}_{2}$ and $\mathrm{y}_{3}$, but never both.

Theorem 3.8 (E-Fritz John necessary optimality conditions for the problem (VP) (3.1)). Let $E: R^{n} \rightarrow R^{n}$ be an one-to-one and onto operator. Let $\bar{x} \in \Omega$ be a weak Pareto solution of the E-vector optimization problem $\left(V P_{\mathrm{E}}\right)$ (3.2) (and, thus, $\mathrm{E}(\overline{\mathrm{x}})$ be a weak E-Pareto solution of the considered multiobjective programming problem $(V P)$ (3.1)). Further, assume that the objective functions $f_{i}, i \in I$, and the constraint functions $g_{j}, j \in J$, are $\mathrm{E}$-differentiable at $\bar{x}$, the constraint functions $\mathrm{h}_{\mathrm{t}}, \mathrm{t} \in \mathrm{T}$, are continuously $\mathrm{E}$-differentiable at $\bar{x}$. If the gradients of equality constraints at $\bar{x}$ are linearly independent, then there exist Lagrange multipliers $\bar{\lambda} \in R^{p}, \bar{\mu} \in R^{m}, \bar{\xi} \in R^{q}$ such that

$$
\begin{gathered}
\sum_{i=1}^{p} \bar{\lambda}_{i} \nabla\left(f_{i} \circ E\right)(\bar{x})+\sum_{j=1}^{m} \bar{\mu}_{j} \nabla\left(g_{j} \circ E\right)(\bar{x})+\sum_{t=1}^{q} \bar{\xi}_{t} \nabla\left(h_{t} \circ E\right)(\bar{x})=0, \\
\bar{\mu}_{j}\left(g_{j} \circ E\right)(\bar{x})=0, j \in J, \\
(\bar{\lambda}, \bar{\mu}) \geqslant 0 .
\end{gathered}
$$

Proof. Let $\bar{x} \in \Omega$ be a weak Pareto solution of the E-vector optimization problem ( $\left.\mathrm{VP}_{\mathrm{E}}\right)$ (3.2). Then, by Lemma 3.5, $E(\bar{x})$ is a weak E-Pareto solution of the considered multiobjective programming problem (VP) (3.1). Now, we prove that there does not exist $d \in R^{n}, d \neq 0$, satisfying the following system of relations:

$$
\begin{gathered}
\nabla\left(f_{i} \circ E\right)(\bar{x})^{\top} d<0, \quad i \in I, \\
\nabla\left(g_{j} \circ E\right)(\bar{x})^{\top} d<0, j \in J_{E}(\bar{x}), \\
\nabla\left(h_{t} \circ E\right)(\bar{x})^{\top} d=0, t \in T .
\end{gathered}
$$

We proceed by contradiction. Suppose, contrary to the result, that there exists $d \in R^{n}, d \neq 0$, satisfying (3.6), (3.7), (3.8). By assumption, $h_{t}, t \in T$, are E-differentiable at $\bar{x}$. Hence, by Definition 2.6, we have

$$
\left(h_{t} \circ E\right)\left(x_{k}\right)=\left(h_{t} \circ E\right)(\bar{x})+\nabla\left(h_{t} \circ E\right)(\bar{x})\left(x_{k}-\bar{x}\right)+\theta_{h_{t}}\left(\bar{x}, x_{k}-\bar{x}\right)\left\|x_{k}-\bar{x}\right\|, t \in T,
$$

where $\theta_{h_{t}}\left(\bar{x}, x_{k}-\bar{x}\right) \rightarrow 0, t \in T$, as $x_{k} \rightarrow \bar{x}$. Using $\left(h_{t} \circ E\right)(\bar{x})=0$ and dividing the above inequalities by $\left\|x_{k}-\bar{x}\right\|$, we obtain

$$
\frac{\left(h_{t} \circ E\right)\left(x_{k}\right)}{\left\|x_{k}-\bar{x}\right\|}=\nabla\left(h_{t} \circ E\right)(\bar{x}) \frac{x_{k}-\bar{x}}{\left\|x_{k}-\bar{x}\right\|}+\theta_{h_{t}}\left(\bar{x}, x_{k}-\bar{x}\right), t \in T .
$$

Since $h_{t}, t \in T$, are continuously E-differentiable at $\bar{x}$ and $\nabla\left(h_{t} \circ E\right)(\bar{x}), t \in T$, are linearly independent, by [35, Proposition 2C], we have that there exists $\widetilde{x}_{k} \rightarrow \bar{x}$ such that $\theta_{h_{t}}\left(\bar{x}, \widetilde{x}_{k}-\bar{x}\right) \rightarrow 0$, $\frac{\widetilde{x}_{k}-\bar{x}}{\left\|\tilde{x}_{k}-\bar{x}\right\|} \rightarrow d$ and, moreover,

$$
\left(h_{\mathrm{t}} \circ \mathrm{E}\right)\left(\widetilde{\mathrm{x}}_{\mathrm{k}}\right)=0, \quad \forall \mathrm{t} \in \mathrm{T} .
$$


By assumption, $g_{j}, j \in J$, are E-differentiable at $\bar{x}$. Hence, by Definition 2.6, we have

$$
\left(g_{j} \circ E\right)\left(\widetilde{x}_{k}\right)=\left(g_{j} \circ E\right)(\bar{x})+\nabla\left(g_{j} \circ E\right)(\bar{x})\left(\widetilde{x}_{k}-\bar{x}\right)+\theta_{g_{j}}\left(\bar{x}, \widetilde{x}_{k}-\bar{x}\right)\left\|\widetilde{x}_{k}-\bar{x}\right\|, j \in J,
$$

where $\theta_{g_{j}}\left(\bar{x}, \widetilde{x}_{k}-\bar{x}\right) \rightarrow 0, j \in J$, as $x_{k} \rightarrow \bar{x}$. Since $\left(g_{j} \circ E\right)(\bar{x})=0, j \in J_{E}(\bar{x})$, and taking $\theta_{g_{j}}\left(\bar{x}, \widetilde{x}_{k}-\bar{x}\right) \rightarrow 0$, $j \in J, \frac{\widetilde{x}_{k}-\bar{x}}{\left\|\widetilde{x}_{k}-\bar{x}\right\|} \rightarrow$ d as $\widetilde{x}_{k} \rightarrow \bar{x}$, inequalities (3.9) yield

$$
\left(g_{j} \circ E\right)\left(\widetilde{x}_{k}\right)<0, j \in J_{E}(\bar{x}) .
$$

By assumption, the objective functions $f_{i}, i \in I$, are E-differentiable at $\bar{x}$. Thus, by Definition 2.6, we have

$$
\left(f_{i} \circ E\right)\left(\widetilde{x}_{k}\right)=\left(f_{i} \circ E\right)(\bar{x})+\nabla\left(f_{i} \circ E\right)(\bar{x})^{\top}\left(\widetilde{x}_{k}-\bar{x}\right)+\theta_{i}\left(\bar{x}, \widetilde{x}_{k}-\bar{x}\right)\left\|\widetilde{x}_{k}-\bar{x}\right\| i \in I .
$$

Using $\theta_{f_{i}}\left(\bar{x}, x_{k}-\bar{x}\right) \rightarrow 0$ and $\frac{x_{k}-\bar{x}}{\left\|x_{k}-\bar{x}\right\|} \rightarrow d$ as $\widetilde{x}_{k} \rightarrow \bar{x}$ together with (3.9), we get that the following inequalities

$$
\left(f_{i} \circ E\right)\left(\widetilde{x}_{k}\right)<\left(f_{i} \circ E\right)(\bar{x}), i \in I
$$

hold, which is a contradiction to the assumption that $\bar{x} \in \Omega$ be a weak Pareto solution of the E-vector optimization problem $\left(\mathrm{VP}_{\mathrm{E}}\right)$ (3.2) (or, in other words, to the assumption that $\mathrm{E}(\overline{\mathrm{x}})$ is a weak E-Pareto solution of the considered multiobjective programming problem (VP) (3.1)). This means that there does not exist any $d \in R^{n}$ satisfying the system of relations (3.6), (3.7), (3.8). Hence, by Motzkin's theorem of the alternative (see Theorem 3.7), there exist $\bar{\lambda}_{i} \in R^{p}, \bar{\mu}_{j}, j \in J\left(E(\bar{x})\right.$ ), and $\bar{\xi} \in R^{q}$ such that

$$
\sum_{i=1}^{p} \bar{\lambda}_{i} \nabla\left(f_{i} \circ E\right)(\bar{x})+\sum_{j \in J_{E}(\bar{x})} \bar{\mu}_{j} \nabla\left(g_{j} \circ E\right)(\bar{x})+\sum_{t=1}^{q} \bar{\xi}_{t} \nabla\left(h_{t} \circ E\right)(\bar{x})=0 .
$$

If we set that $\bar{\mu}_{j}=0$ for all $j \in J \backslash J_{E}(\bar{x})$, then (3.10) implies (3.3). Further, note that also the complementary slackness condition (3.4) is satisfied. Indeed, if $g_{j}(E(\bar{x}))<0$, then $j \in J \backslash J_{E}(\bar{x})$ and $\bar{\mu}_{j}=0$. The proof of this theorem is completed.

Before we prove the Karush-Kuhn-Tucker necessary optimality conditions for the differentiable Evector optimization problem (VP) (3.1) and, thus, the E-Karush-Kuhn-Tucker necessary optimality conditions for the considered E-differentiable vector optimization problem (VP) (3.1), we introduce the so-called E-Abadie constraint qualification. In order to do this, for the E-vector optimization problem $\left(\mathrm{VP}_{\mathrm{E}}\right)(3.2)$, we introduce two different linear approximations of the feasible set $\Omega_{E}$. Namely, the first of them is the E-linearized cone $L_{E}(\bar{x})$ and the second one is the tangent cone $T_{\Omega_{E}}(\bar{x})$.

Definition 3.9. For the $E$-vector optimization problem $\left(\mathrm{VP}_{E}\right)$ (3.2), the E-linearized cone at $\bar{x} \in \Omega_{E}$, denoted by $\mathrm{L}_{E}(\bar{x})$, is defined as follows

$$
\mathrm{L}_{E}(\bar{x})=\left\{\mathrm{d} \in \mathrm{R}^{\mathrm{n}}: \nabla \mathrm{g}_{j}(\mathrm{E}(\overline{\mathrm{x}})) \mathrm{d} \leqq 0, j \in \mathrm{J}_{\mathrm{E}}(\overline{\mathrm{x}}), \nabla \mathrm{h}_{\mathrm{t}}(\mathrm{E}(\overline{\mathrm{x}})) \mathrm{d}=0, \mathrm{t} \in \mathrm{T}\right\} .
$$

It is easy to see that $L_{E}(\bar{x})$ is a nonempty closed convex cone.

Now, for the E-vector optimization problem $\left(\mathrm{VP}_{\mathrm{E}}\right)$ (3.2), we define a linear approximation of the feasible set $\Omega_{\mathrm{E}}$ by the help of tangent directions, in other words, we define the tangent cone to the feasible set $\Omega_{\mathrm{E}}$.

Definition 3.10. The tangent cone (also called contingent cone or Bouligand cone) of $\Omega_{E}$ at $\bar{x} \in \operatorname{cl} \Omega_{E}$ is defined by

$$
\mathrm{T}_{\Omega_{\mathrm{E}}}(\bar{x})=\left\{\mathrm{d} \in \mathrm{R}^{\mathrm{n}}: \exists_{\left\{\mathrm{d}_{\mathrm{n}}\right\} \subset \mathrm{R}^{n}} \mathrm{~d}_{\mathrm{n}} \rightarrow \mathrm{d}, \exists_{\left\{\mathrm{t}_{\mathrm{n}}\right\} \subset \mathrm{R}} \mathrm{t}_{\mathrm{n}} \downarrow 0 \text { s.t. } \bar{x}+\mathrm{t}_{\mathrm{n}} \mathrm{d}_{\mathrm{n}} \in \Omega_{\mathrm{E}}\right\},
$$

or, equivalently,

$$
\mathrm{T}_{\Omega_{\mathrm{E}}}(\bar{x})=\left\{\mathrm{d} \in \mathrm{R}^{\mathrm{n}}: \exists_{\left\{\beta_{n}\right\} \subset \mathrm{R}} \beta_{\mathrm{n}} \rightarrow \infty, \exists_{\left\{x_{n}\right\} \subset \Omega_{\mathrm{E}}} x_{\mathrm{n}} \rightarrow \bar{x} \text { s.t. } \beta_{n}\left(x_{n}-\bar{x}\right) \rightarrow \mathrm{d}\right\} .
$$

A vector $d \in R^{n}$ belonging to $T_{\Omega_{E}}(\bar{x})$ is called a tangent direction to $\Omega_{E}$ from $\bar{x} \in \operatorname{cl} \Omega_{E}$. 
Remark 3.11. Note that this cone is always closed and it is convex if the original set is so.

Now, we prove that, for any $\bar{x} \in \Omega_{E}, T_{\Omega_{E}}(\bar{x})$ is always a subset of $L_{E}(\bar{x})$.

Proposition 3.12. For any $\bar{x} \in \Omega_{E}, T_{\Omega_{E}}(\bar{x}) \subset L_{E}(\bar{x})$.

Proof. Let $\bar{x} \in \Omega_{E}$ be given and let $d$ be any nonzero vector belonging to $T_{\Omega_{E}}(\bar{x})$. Then, by Definition 3.10, there exists a sequence $\left(x_{k}\right) \subset \Omega_{E}$ such that $x_{k} \rightarrow \bar{x}$ and $\frac{x_{k}-\bar{x}}{\left\|x_{k}-\bar{x}\right\|} \rightarrow d$. By assumption, all constraint functions $g_{j}, j \in J$ and $h_{t}, t \in T$, are E-differentiable at $\bar{x}$. Hence, by Definition 2.6, it follows that

$$
\begin{aligned}
& \left(g_{j} \circ E\right)\left(x_{k}\right)=\left(g_{j} \circ E\right)(\bar{x})+\nabla\left(g_{j} \circ E\right)(\bar{x})\left(x_{k}-\bar{x}\right)+\theta_{g_{j}}\left(\bar{x}, x_{k}-\bar{x}\right)\left\|x_{k}-\bar{x}\right\|, j \in J, \\
& \left(h_{t} \circ E\right)\left(x_{k}\right)=\left(h_{t} \circ E\right)(\bar{x})+\nabla\left(h_{t} \circ E\right)(\bar{x})\left(x_{k}-\bar{x}\right)+\theta_{h_{t}}\left(\bar{x}, x_{k}-\bar{x}\right)\left\|x_{k}-\bar{x}\right\|, t \in T,
\end{aligned}
$$

where $\theta_{g_{j}}\left(\bar{x}, x_{k}-\bar{x}\right) \rightarrow 0, j \in J, \theta_{h_{t}}\left(\bar{x}, x_{k}-\bar{x}\right) \rightarrow 0, t \in T$, as $x_{k} \rightarrow \bar{x}$. Since $x_{k} \subset \Omega_{E}$ for each $k \in N$ and $\bar{x} \in \Omega_{\mathrm{E}}$, we have, respectively,

$$
\begin{gathered}
\nabla g_{j}(E(\bar{x}))\left(x_{k}-\bar{x}\right)+\theta_{g_{j}}\left(\bar{x}, x_{k}-\bar{x}\right)\left\|x_{k}-\bar{x}\right\| \leqq 0, j \in J_{E}(\bar{x}), \\
\nabla h_{t}(E(\bar{x}))\left(x_{k}-\bar{x}\right)+\theta_{h_{t}}\left(\bar{x}, x_{k}-\bar{x}\right)\left\|x_{k}-\bar{x}\right\|=0, t \in T .
\end{gathered}
$$

Then, dividing the above inequalities by $\left\|x_{k}-\bar{x}\right\|$, we obtain, respectively,

$$
\begin{gathered}
\nabla g_{j}(E(\bar{x})) \frac{x_{k}-\bar{x}}{\left\|x_{k}-\bar{x}\right\|}+\theta_{g_{j}}\left(\bar{x}, x_{k}-\bar{x}\right) \leqq 0, j \in J_{E}(\bar{x}), \\
\nabla h_{t}(E(\bar{x})) \frac{x_{k}-\bar{x}}{\left\|x_{k}-\bar{x}\right\|}+\theta_{h_{t}}\left(\bar{x}, x_{k}-\bar{x}\right)=0, t \in T .
\end{gathered}
$$

Taking now limits $\theta_{g_{j}}\left(\bar{x}, x_{k}-\bar{x}\right) \rightarrow 0, j \in J, \theta_{h_{t}}\left(\bar{x}, x_{k}-\bar{x}\right) \rightarrow 0, t \in T$, as $x_{k} \rightarrow \bar{x}$ and $\frac{x_{k}-\bar{x}}{\left\|x_{k}-\bar{x}\right\|} \rightarrow d$, we get, respectively,

$$
\begin{gathered}
\nabla g_{j}(E(\bar{x})) d \leqq 0, j \in J_{E}(\bar{x}), \\
\nabla h_{t}(E(\bar{x})) d=0, t \in T .
\end{gathered}
$$

By (3.11), (3.12) and Definition 3.9, it follows that $d \in L_{E}(\bar{x})$. Hence, the proof of this proposition is completed.

Note that the converse result is not true. Namely, the sets $L_{E}(\bar{x})$ and $T_{\Omega_{E}}(\bar{x})$ do not coincide in general. In order to illustrate this fact, we present the following example of an E-differentiable vector optimization problem for which we construct its associated E-vector optimization problem.

Example 3.13. Consider the following nondifferentiable vector optimization problem

$$
\begin{aligned}
& f\left(x_{1}, x_{2}\right)=\left(\sqrt[3]{x_{1}}+\sqrt[3]{x_{2}^{2}}, \sqrt[3]{x_{1}^{2}}+\sqrt[3]{x_{2}}\right) \rightarrow \min \\
& g_{1}\left(x_{1}, x_{2}\right)=\sqrt[3]{x_{1}}-\sqrt[3]{x_{2}} \leqq 0 \\
& h_{1}\left(x_{1}, x_{2}\right)=\sqrt[3]{x_{1} x_{2}}=0
\end{aligned}
$$

Note that $\Omega=\left\{\left(x_{1}, x_{2}\right) \in R^{2}: \sqrt[3]{x_{1}}-\sqrt[3]{x_{2}} \leqq 0 \wedge \sqrt[3]{x_{1} x_{2}}=0\right\}$. Let $E: R^{2} \rightarrow R^{2}$ be an operator defined by $E\left(x_{1}, x_{2}\right)=\left(x_{1}^{3}, x_{2}^{3}\right)$. For the considered vector optimization problem (VP1) (3.13), we define its associated E-vector optimization problem $\left(\mathrm{VP}_{\mathrm{E}}\right)$ (3.2) as follows

$$
\begin{aligned}
& f\left(E\left(x_{1}, x_{2}\right)\right)=\left(x_{1}+x_{2}^{2}, x_{1}^{2}+x_{2}\right) \rightarrow \min \\
& g_{1}\left(E\left(x_{1}, x_{2}\right)\right)=x_{1}-x_{2} \leqq 0, \\
& h_{1}\left(E\left(x_{1}, x_{2}\right)\right)=x_{1} x_{2}=0 .
\end{aligned}
$$

Then, $\Omega_{\mathrm{E}}=\left\{\left(x_{1}, x_{2}\right) \in R^{2}: x_{1}-x_{2} \leqq 0 \wedge x_{1} x_{2}=0\right\}$ and $\bar{x}=(0,0)$ is a feasible solution. Then, by Definition 3.10, we have that $T_{\Omega_{E}}(\bar{x})=\left\{d \in R^{2}: d_{1} \leqq 0, d_{2} \geqq 0, d_{1} d_{2}=0\right\}$. Further, by Definition 3.9, we have that $L_{E}(\bar{x})=\left\{d \in R^{2}: d_{1}-d_{2} \leqq 0\right\}$. Then, $L_{E}(\bar{x}) \nsubseteq T_{\Omega_{E}}(\bar{x})$. 
Proposition 3.14. Let $\mathrm{f}: \mathrm{R}^{\mathrm{n}} \rightarrow \mathrm{R}$ be an $\mathrm{E}$-differentiable function at $\overline{\mathrm{x}} \in \Omega_{\mathrm{E}}$. If $\overline{\mathrm{x}}$ is a weak Pareto solution of the $\mathrm{E}-$-vector optimization problem $\left(V P_{\mathrm{E}}\right)(3.2)$ (and, thus, $\mathrm{E}(\overline{\mathrm{x}})$ is a weak $\mathrm{E}$-Pareto of the considered multiobjective programming problem $(V P)(3.1))$, then $\nabla(\mathrm{f} \circ \mathrm{E})(\bar{x}) \mathrm{d} \nless 0$ for each $\mathrm{d} \in \mathrm{T}_{\Omega_{\mathrm{E}}}(\overline{\mathrm{x}})$. In other words, this means that the system inequalities $\nabla\left(f_{i} \circ \mathrm{E}\right)(\bar{x}) \mathrm{d}<0, i \in \mathrm{I}$, has no any solution $\mathrm{d} \in \mathrm{T}_{\Omega_{\mathrm{E}}}(\overline{\mathrm{x}})$, that is, it is satisfied $\max _{i=1, \cdots, p}\left\{\nabla\left(f_{i} \circ E\right)(\bar{x}) d\right\} \geqq 0$ for each $d \in T_{\Omega_{E}}(\bar{x})$.

Proof. Let $f: R^{n} \rightarrow R$ be an E-differentiable function at $\bar{x} \in \Omega_{E}$. Hence, by Definition 2.6, we have

$$
\left(f_{i} \circ E\right)\left(x_{k}\right)=\left(f_{i} \circ E\right)(\bar{x})+\nabla\left(f_{i} \circ E\right)(\bar{x})\left(x_{k}-\bar{x}\right)+\theta_{f_{i}}\left(\bar{x}, x_{k}-\bar{x}\right)\left\|x_{k}-\bar{x}\right\|, i \in I,
$$

where $\theta_{f_{i}}\left(\bar{x}, x_{k}-\bar{x}\right) \rightarrow 0, i \in I$, as $x_{k} \rightarrow \bar{x}$. Further, assume that $\bar{x}$ is a weak Pareto solution of the E-vector optimization problem $\left(\mathrm{VP}_{\mathrm{E}}\right)$ (3.2). Suppose, contrary to the result, that there exists $d \in \mathrm{T}_{\Omega_{\mathrm{E}}}(\bar{x}), d \neq 0$, such that $\nabla(f \circ E)(\bar{x}) d<0$. Then, (3.14) gives

$$
\left(f_{i} \circ E\right)\left(x_{k}\right)-\left(f_{i} \circ E\right)(\bar{x})<\theta_{f_{i}}\left(\bar{x}, x_{k}-\bar{x}\right)\left\|x_{k}-\bar{x}\right\|, i \in I .
$$

Taking now limits $\theta_{f_{i}}\left(\bar{x}, x_{k}-\bar{x}\right) \rightarrow 0, i \in I$, as $x_{k} \rightarrow \bar{x}$, we get that the inequalities

$$
\left(f_{i} \circ E\right)\left(x_{k}\right)-\left(f_{i} \circ E\right)(\bar{x})<0, \quad i \in I
$$

hold for $k$ large enough, which is a contradiction to the assumption that $\bar{x}$ is a weak Pareto solution of the E-vector optimization problem $\left(\mathrm{VP}_{\mathrm{E}}\right)$ (3.2).

Now, we prove the Karush-Kuhn-Tucker necessary optimality conditions for the differentiable E-vector optimization problem ( $\left.\mathrm{VP}_{\mathrm{E}}\right)$ (3.2) and, thus, the so-called E-Karush-Kuhn-Tucker necessary optimality conditions for not necessarily differentiable multiobjective programming problem (VP) (3.1) in which the involved functions are E-differentiable.

In order to prove the E-Karush-Kuhn-Tucker necessary optimality conditions for a weak E-Pareto solution of the E-differentiable multiobjective programming problem (VP) (3.1), we introduce the so-called E-Abadie constraint qualification for the differentiable vector optimization problem $\left(\mathrm{VP}_{\mathrm{E}}\right)(3.2)$ with both inequality and equality constraints.

Definition 3.15. It is said that the so-called $E$-Abadie constraint qualification (ACQE) holds at $\bar{x} \in \Omega_{E}$ for the differentiable $E$-vector optimization problem $\left(\mathrm{VP}_{\mathrm{E}}\right)$ (3.2) with both inequality and equality constraints if

$$
\mathrm{T}_{\Omega_{\mathrm{E}}}(\bar{x})=\mathrm{L}_{\mathrm{E}}(\overline{\mathrm{x}}) .
$$

Theorem 3.16 (E-Karush-Kuhn-Tucker necessary optimality conditions). Let $\bar{x} \in \Omega_{\mathrm{E}}$ be a weak Pareto solution of the $\mathrm{E}$-vector optimization problem $\left(V P_{\mathrm{E}}\right)(3.2)$ (and, thus, $\mathrm{E}(\overline{\mathrm{x}})$ be a weak $\mathrm{E}-$ Pareto solution of the considered multiobjective programming problem (VP) (3.1)). Further, let the objective functions $f_{i}, i \in I$, the constraint functions $\mathrm{g}_{\mathrm{j}}, \mathrm{j} \in \mathrm{J}$, and $\mathrm{h}_{\mathrm{t}}, \mathrm{t} \in \mathrm{T}$, be $\mathrm{E}$-differentiable at $\overline{\mathrm{x}}$ and the $\mathrm{E}$-Abadie constraint qualification (ACQ $\mathrm{E}$ ) be satisfied at $\bar{\chi}$. Then there exist Lagrange multipliers $\bar{\lambda} \in R^{p}, \bar{\mu} \in R^{m}$ and $\bar{\xi} \in R^{q}$ such that

$$
\begin{gathered}
\sum_{i=1}^{p} \bar{\lambda}_{i} \nabla\left(f_{i} \circ E\right)(\bar{x})+\sum_{j=1}^{m} \bar{\mu}_{j} \nabla\left(g_{j} \circ E\right)(\bar{x})+\sum_{t=1}^{q} \bar{\xi}_{t} \nabla\left(h_{t} \circ E\right)(\bar{x})=0, \\
\bar{\mu}_{j}\left(g_{j} \circ E\right)(\bar{x})=0, j \in J, \\
\bar{\lambda} \geqslant 0, \bar{\mu} \geqq 0 .
\end{gathered}
$$

Proof. Let $\bar{x} \in \Omega$ be a weak Pareto solution of the differentiable E-vector optimization problem (VP $\mathrm{E}_{\mathrm{E}}$ (3.2) (and, thus, $E(\bar{x})$ be a weak E-Pareto solution of the considered multiobjective programming problem (VP) (3.1)). We show that the system

$$
\nabla\left(f_{i} \circ E\right)(\bar{x})^{\top} d<0, \quad i \in I,
$$




$$
\begin{gathered}
\nabla\left(g_{j} \circ E\right)(\bar{x})^{\top} d \leqq 0, j \in J_{E}(\bar{x}), \\
\nabla\left(h_{t} \circ E\right)(\bar{x})^{\top} d=0, t \in T
\end{gathered}
$$

has no any solution $d \in R^{n}$. Suppose, contrary to the result, that the system (3.19), (3.20), (3.21) has a solution $d \in R^{n}$. By assumption, the E-Abadie constraint qualification (ACQE) is satisfied at $\bar{x}$. Then, by (3.15), it follows that $d \in T_{\Omega_{E}}(\bar{x})$. By Proposition 3.14, it follows that the following relation $\max _{i=1, \cdots, p}\left\{\nabla\left(f_{i} \circ E\right)(\bar{x}) d\right\} \geqq 0$ holds for each $d \in T_{\Omega_{E}}(\bar{x})$, which contradicting (3.19). Since the system (3.19), (3.20), (3.21) has no any solution $d \in R^{n}$, by Motzkin theorem (Theorem 3.7), there exist Lagrange multipliers $\bar{\lambda} \in R^{p}, \bar{\mu} \in R^{m}$ and $\bar{\xi} \in R^{q}$ such that the conditions (3.16), (3.17), (3.18) are fulfilled by setting $\bar{\mu}_{j}=0$ for all $j \in J \backslash J_{E}(\bar{x})$. Thus, the proof of this theorem is completed.

In order to illustrate the above result, we present an example of such a nondifferentiable vector optimization problem for which the E-Abadie constraint qualification is not satisfied. Note that, in such a case, Lagrange multiplier $\bar{\lambda}$ corresponding to the objective function $f$ can be equal 0 .

Example 3.17. We consider again the nondifferentiable vector optimization problem (VP1) (3.13) considered in Example 3.13. Note that $\bar{x}=(0,0)$ is a Pareto solution of the E-vector optimization problem constructed and, thus, it is also a E-Pareto solution of the nondifferentiable multiobjective programming problem (VP1) (3.13). However, as it follows from Example 3.13, the E-Abadie constraint qualification $\left(A C Q_{E}\right)$ does not hold at $\bar{x} \in \Omega_{\mathrm{E}}$. Now, we show that E-Karush-Kuhn-Tucker necessary optimality conditions are not satisfied at such a case. Indeed, as it follows from the conditions (3.16), (3.17), (3.18), the following equations $\bar{\lambda}_{1}+\bar{\mu}_{1}=0$ and $\bar{\lambda}_{2}-\bar{\mu}_{1}=0$ are fulfilled only in the case when $\bar{\lambda}_{1}=0$ and $\bar{\lambda}_{2}=0$, what is impossible.

Definition 3.18. $(\bar{x}, \bar{\lambda}, \bar{\mu}, \bar{\xi}) \in \Omega_{E} \times R^{p} \times R^{m} \times R^{q}$ is said to be a Karush-Kuhn-Tucker point for the vector optimization problem $\left(\mathrm{VP}_{\mathrm{E}}\right)$ (3.2) if the Karush-Kuhn-Tucker necessary optimality conditions (3.16), (3.17), (3.18) are satisfied at $\bar{\chi}$ with Lagrange multiplier $\bar{\lambda}, \bar{\mu}, \bar{\xi}$.

Now, we prove the sufficiency of the E-Karush-Kuhn-Tucker necessary optimality conditions for the considered E-differentiable vector optimization problem (VP) (3.1) under E-convexity hypotheses.

Theorem 3.19. Let $(\bar{x}, \bar{\lambda}, \bar{\mu}, \bar{\xi}) \in \Omega \times R^{p} \times R^{m} \times R^{q}$ be a Karush-Kuhn-Tucker point of the E-vector optimization problem $\left(V P_{\mathrm{E}}\right)$ (3.2). Let $\mathrm{T}_{\mathrm{E}}^{+}(\mathrm{E}(\overline{\mathrm{x}}))=\left\{\mathrm{t} \in \mathrm{T}: \bar{\xi}_{\mathrm{t}}>0\right\}$ and $\mathrm{T}_{\mathrm{E}}^{-}(\mathrm{E}(\overline{\mathrm{x}}))=\left\{\mathrm{t} \in \mathrm{T}: \bar{\xi}_{\mathrm{t}}<0\right\}$. Furthermore, assume the following hypotheses are fulfilled:

(a) each objective function $\mathrm{f}_{\mathrm{i}}, \mathrm{i} \in \mathrm{I}$, is an $\mathrm{E}$-convex function at $\overline{\mathrm{x}}$ on $\Omega_{\mathrm{E}}$;

(b) each inequality constraint $\mathrm{g}_{\mathrm{j}}, \mathrm{j} \in \mathrm{J}_{\mathrm{E}}(\overline{\mathrm{x}})$, is an $\mathrm{E}$-convex function at $\overline{\mathrm{x}}$ on $\Omega_{\mathrm{E}}$;

(c) each equality constraint $h_{t}, t \in T^{+}(\mathrm{E}(\bar{x}))$, is an $\mathrm{E}$-convex function at $\bar{x}$ on $\Omega_{\mathrm{E}}$;

(d) each function $-h_{\mathrm{t}}, \mathrm{t} \in \mathrm{T}^{-}(\mathrm{E}(\overline{\mathrm{x}}))$, is an $\mathrm{E}$-convex function at $\overline{\mathrm{x}}$ on $\Omega_{\mathrm{E}}$.

Then $\bar{x}$ is a weak Pareto solution of the problem (VP $\mathrm{E})(3.2)$ and, thus, $\mathrm{E}(\overline{\mathrm{x}})$ is a weak $\mathrm{E}-$ Pareto solution of the problem $(V P)(3.1)$.

Proof. By assumption, $(\bar{x}, \bar{\lambda}, \bar{\mu}, \bar{\xi}) \in \Omega \times R^{p} \times R^{m} \times R^{q}$ is a Karush-Kuhn-Tucker point of the differentiable vector optimization problem (VP $\mathrm{VP}_{\mathrm{E}}$ ) (3.2). Then, by Definition 3.18, the Karush-Kuhn-Tucker necessary optimality conditions (3.16), (3.17), (3.18) are satisfied at $\bar{x}$ with Lagrange multipliers $\bar{\lambda} \in R^{p}, \bar{\mu} \in R^{m}$ and $\bar{\xi} \in R^{q}$. We proceed by contradiction. Suppose, contrary to the result, that $\bar{x}$ is not a weak Pareto solution of the problem $\left(\mathrm{VP}_{\mathrm{E}}\right)$ (3.2). Hence, by Definition 3.1, there exists another $\widetilde{x} \in \Omega_{\mathrm{E}}$ such that

$$
f(E(\widetilde{x}))<f(E(\bar{x})) .
$$

Using hypotheses (a)-(d), by Proposition 2.7 and Definition 2.2, the following inequalities

$$
f_{i}(E(\widetilde{x}))-f_{i}(E(\bar{x})) \geqq \nabla f_{i}(E(\bar{x}))(E(\widetilde{x})-E(\bar{x})), i \in I,
$$




$$
\begin{gathered}
g_{j}(E(\widetilde{x}))-g_{j}(E(\bar{x})) \geqq \nabla g_{j}(E(\bar{x}))(E(\widetilde{x})-E(\bar{x})), j \in J(E(\bar{x})), \\
h_{t}(E(\widetilde{x}))-h_{t}(E(\bar{x})) \geqq \nabla h_{t}(E(\bar{x}))(E(\widetilde{x})-E(\bar{x})), t \in T^{+}(E(\bar{x})), \\
-h_{t}(E(\widetilde{x}))+h_{t}(E(\bar{x})) \geqq-\nabla h_{t}(E(\bar{x}))(E(\widetilde{x})-E(\bar{x})), t \in T^{-}(E(\bar{x}))
\end{gathered}
$$

hold, respectively. Combining (3.22) and (3.23) and then multiplying the resulting inequalities by the corresponding Lagrange multipliers and adding both their sides, we get

$$
\left[\sum_{i=1}^{p} \bar{\lambda}_{i} \nabla\left(f_{i} \circ E\right)(\bar{x})\right](E(\widetilde{x})-E(\bar{x}))<0 .
$$

Multiplying inequalities (3.24), (3.25), (3.26) by the corresponding Lagrange multipliers, respectively, we obtain

$$
\begin{gathered}
\bar{\mu}_{j} g_{j}(E(\widetilde{x}))-\bar{\mu}_{j} g_{j}(E(\bar{x})) \geqq \bar{\mu}_{j} \nabla g_{j}(E(\bar{x}))(E(\widetilde{x})-E(\bar{x})), j \in J(E(\bar{x})), \\
\bar{\xi}_{t} h_{t}(E(\widetilde{x}))-\bar{\xi}_{t} h_{t}(E(\bar{x})) \geqq \bar{\xi}_{t} \nabla h_{t}(E(\bar{x}))(E(\widetilde{x})-E(\bar{x})), t \in T^{+}(E(\bar{x})), \\
\bar{\xi}_{t} h_{t}(E(\widetilde{x}))-\bar{\xi}_{t} h_{t}(E(\bar{x})) \geqq \bar{\xi}_{t} \nabla h_{t}(E(\bar{x}))(E(\widetilde{x})-E(\bar{x})), t \in T^{-}(E(\bar{x})) .
\end{gathered}
$$

Using the E-Karush-Kuhn-Tucker necessary optimality condition (3.17) together with $\widetilde{x} \in \Omega_{E}$ and $\bar{x} \in \Omega_{E}$, we get, respectively,

$$
\begin{gathered}
\bar{\mu}_{j} \nabla g_{j}(E(\bar{x}))(E(\widetilde{x})-E(\bar{x})) \leqq 0, j \in J(E(\bar{x})), \\
\bar{\xi}_{t} \nabla h_{t}(E(\bar{x}))(E(\widetilde{x})-E(\bar{x})) \leqq 0, t \in T^{+}(E(\bar{x})), \\
\bar{\xi}_{t} \nabla h_{t}(E(\bar{x}))(E(\widetilde{x})-E(\bar{x})) \leqq 0, t \in T^{-}(E(\bar{x})) .
\end{gathered}
$$

Combining (3.27) and (3.28), (3.29), (3.30), we obtain that the following inequality

$$
\left[\sum_{i=1}^{p} \bar{\lambda}_{i} \nabla\left(f_{i} \circ E\right)(\bar{x})+\sum_{j=1}^{m} \bar{\mu}_{j} \nabla g_{j}(E(\bar{x}))+\sum_{t=1}^{q} \bar{\mu}_{t} \nabla h_{t}(E(\bar{x}))\right](E(\widetilde{z})-E(\bar{x}))<0
$$

holds, which is a contradiction to the E-Karush-Kuhn-Tucker necessary optimality condition (3.16). By assumption, $E: R^{n} \rightarrow R^{n}$ is an one-to-one and onto operator. Since $\bar{x}$ is a weak Pareto solution of the problem $\left(\mathrm{VP}_{\mathrm{E}}\right)(3.2)$, by Lemma 3.5, $\mathrm{E}(\overline{\mathrm{x}})$ is a weak E-Pareto solution of the problem (VP) (3.1). Thus, the proof of this theorem is completed.

Theorem 3.20. Let $(\bar{x}, \bar{\lambda}, \bar{\mu}, \bar{\xi}) \in \Omega \times \mathrm{R}^{\mathrm{p}} \times \mathrm{R}^{\mathrm{m}} \times \mathrm{R}^{\mathrm{q}}$ be a Karush-Kuhn-Tucker point of the E-vector optimization problem $\left(V P_{\mathrm{E}}\right)$ (3.2). Furthermore, assume that the following hypotheses are fulfilled:

(a) each objective function $f_{i}, i \in I$, is a strictly $E$-convex function at $\bar{x}$ on $\Omega_{\mathrm{E}}$;

(b) each inequality constraint $g_{j}, j \in \mathrm{J}_{\mathrm{E}}(\overline{\mathrm{x}})$, is an $\mathrm{E}$-convex function at $\overline{\mathrm{x}}$ on $\Omega_{\mathrm{E}}$;

(c) each equality constraint $h_{t}, t \in T^{+}(E(\bar{x}))$, is an $\mathrm{E}$-convex function at $\bar{x}$ on $\Omega_{\mathrm{E}}$;

(d) each function $-\mathrm{h}_{\mathrm{t}}, \mathrm{t} \in \mathrm{T}^{-}(\mathrm{E}(\overline{\mathrm{x}}))$, is an $\mathrm{E}$-convex function at $\overline{\mathrm{x}}$ on $\Omega_{\mathrm{E}}$.

Then $\bar{x}$ is a Pareto solution of the problem $\left(V P_{\mathrm{E}}\right)(3.2)$ and, thus, $\mathrm{E}(\overline{\mathrm{x}})$ is an $\mathrm{E}-$ Pareto solution of the problem $(V P)$ (3.1).

Remark 3.21. As it follows from the proof of Theorem 3.19, the sufficient conditions are also satisfied if all or some of the functions $g_{j}, j \in J_{E}(\bar{x}), h_{t}, t \in T^{+}(E(\bar{x})),-h_{t}, t \in T^{-}(E(\bar{x}))$, are E-differentiable quasi E-convex function at $\bar{x}$ on $\Omega_{\mathrm{E}}$.

Note that the sufficient optimality conditions for a weak E-Pareto solution of the problem (VP) (3.1) can be established also under generalized E-convexity hypotheses.

Theorem 3.22. Let $(\bar{x}, \bar{\lambda}, \bar{\mu}, \bar{\xi}) \in \Omega \times R^{p} \times R^{m} \times R^{q}$ be a Karush-Kuhn-Tucker point of the E-vector optimization problem $\left(V P_{\mathrm{E}}\right)$ (3.2). Furthermore, assume that the following hypotheses are fulfilled: 
(a) the objective function $\mathrm{f}$ is a pseudo $\mathrm{E}$-convex function at $\overline{\mathrm{x}}$ on $\Omega_{\mathrm{E}}$;

(b) each inequality constraint $\mathrm{g}_{\mathrm{j}}, \mathrm{j} \in \mathrm{J}_{\mathrm{E}}(\overline{\mathrm{x}})$, is a quasi $\mathrm{E}$-convex function at $\overline{\mathrm{x}}$ on $\Omega_{\mathrm{E}}$;

(c) each equality constraint $\mathrm{h}_{\mathrm{t}}, \mathrm{t} \in \mathrm{T}^{+}(\mathrm{E}(\overline{\mathrm{x}}))$, is a quasi $\mathrm{E}$-convex function at $\overline{\mathrm{x}}$ on $\Omega_{\mathrm{E}}$;

(d) each function $-\mathrm{h}_{\mathrm{t}}, \mathrm{t} \in \mathrm{T}^{-}(\mathrm{E}(\overline{\mathrm{x}}))$, is a quasi $\mathrm{E}$-convex function at $\overline{\mathrm{x}}$ on $\Omega_{\mathrm{E}}$.

Then $\bar{x}$ is a weak Pareto solution of the problem (VP $\mathrm{E})(3.2)$ and, thus, $\mathrm{E}(\overline{\mathrm{x}})$ is a weak $\mathrm{E}-$ Pareto solution of the problem $(V P)(3.1)$.

In order to illustrate the sufficient optimality conditions established in the paper, we now present an example of an E-differentiable vector optimization problem in which the involved functions are (generalized) E-convex.

Example 3.23. Consider the following nonconvex nondifferentiable vector optimization problem

$$
\begin{gathered}
\operatorname{minimize} f(x)=\left(\frac{1}{4} x_{1}^{2}+\frac{1}{2} x_{1}+x_{2}^{\frac{1}{3}}, \frac{1}{2} x_{1}-\left(1-\frac{1}{2} x_{1}\right) x_{2}^{\frac{1}{3}}\right) \\
\text { s.t. } g_{1}(x)=x_{2}^{\frac{1}{3}}-\frac{1}{2} x_{1} \leqq 0 \\
g_{2}(x)=-x_{1} x_{2} \leqq 0 \\
h_{1}(x)=x_{2}^{\frac{1}{3}}-\frac{1}{4} x_{1}^{2}=0
\end{gathered}
$$

Note that $\Omega=\left\{\left(x_{1}, x_{2}\right) \in R^{2}: x_{2}^{\frac{1}{3}}-\frac{1}{2} x_{1} \leqq 0 \wedge x_{1} x_{2} \geqq 0 \wedge x_{2}^{\frac{1}{3}}-\frac{1}{4} x_{1}^{2}=0\right\}$. Let $E: R^{2} \rightarrow R^{2}$ be an oneto-one and onto mapping defined as follows $E\left(x_{1}, x_{2}\right)=\left(2 x_{1}, x_{2}^{3}\right)$. Now, for the considered nonconvex nondifferentiable multiobjective programming problem (VP2) (3.31), we define its associated E-vector optimization problem (VP2 $\mathrm{E})$ as follows

$$
\begin{gathered}
\text { minimize } f(E(x))=\left(x_{1}^{2}+x_{1}+x_{2}, x_{1}-\left(1-x_{1}\right) x_{2}\right) \\
\text { s.t. } g_{1}(E(x))=x_{2}-x_{1} \leqq 0 \\
g_{2}(E(x))=-2 x_{1} x_{2}^{3} \leqq 0 \\
h_{1}(E(x))=x_{2}-x_{1}^{2}=0
\end{gathered}
$$

Note that $\Omega_{E}=\left\{\left(x_{1}, x_{2}\right) \in R^{2}: x_{2}-x_{1} \leqq 0 \wedge 2 x_{1} x_{2}^{3} \geqq 0 \wedge x_{2}-x_{1}^{2}=0\right\}$ and $\bar{x}=(0,0)$ is a feasible solution of the problem (VP2 $\mathrm{E}$ ) (3.32). Further, note that all functions constituting the considered vector optimization problem (VP2) (3.31) are E-differentiable at $\bar{x}=(0,0)$. Then, it can also be shown that the E-Karush-Kuhn-Tucker necessary optimality conditions (3.16), (3.17), (3.18) are fulfilled at $\bar{x}=(0,0)$ with Lagrange multipliers $\bar{\lambda}_{1}=\frac{1}{2}, \bar{\lambda}_{2}=\frac{1}{2}, \bar{\mu}=1$ and $\bar{\xi}_{1}=-1$. Further, it can be proved that $f$ is a strictly E-convex function at $\bar{x}$ on $\Omega_{\mathrm{E}}$, the constraint function $g_{1}$ is $\mathrm{E}$-convex at $\bar{x}$ on $\Omega_{\mathrm{E}}$, the constraint function $\mathrm{g}_{2}$ is a quasi E-convex function at $\bar{x}$ on $\Omega_{\mathrm{E}}$, the function $-\mathrm{h}_{1}$ is $\mathrm{E}$-convex at $\bar{x}$ on $\Omega_{\mathrm{E}}$. Hence, by Theorem 3.20 (see also Remark 3.21), $\bar{x}=(0,0)$ is a Pareto solution of the E-vector optimization problem (VP2 $\mathrm{E}$ ) (3.32) and, thus, by Lemma 3.5, $E(\bar{x})$ is an E-Pareto solution of the considered E-differentiable multiobjective programming problem (VP2) (3.31).

\section{E-Wolfe duality}

In this section, a vector dual problem in the sense of Wolfe is considered for the class of E-differentiable vector optimization problems with inequality and equality constraints. Let $E: R^{n} \rightarrow R^{n}$ be a given oneto-one and onto operator. Consider the following dual problem in the sense of Wolfe related to the considered vector optimization problem (VP) (3.1): 


$$
\begin{gathered}
\operatorname{maximize} \psi_{E}(y, \mu, \xi)=(f \circ E)(y)+\left[\sum_{j=1}^{m} \mu_{j}\left(g_{j} \circ E\right)(y)+\sum_{t=1}^{q} \xi_{t}\left(h_{t} \circ E\right)(y)\right] e \\
\text { s.t. } \sum_{i=1}^{p} \lambda_{i} \nabla\left(f_{i} \circ E\right)(y)+\sum_{j=1}^{m} \mu_{j} \nabla\left(g_{j} \circ E\right)(y)+\sum_{t=1}^{q} \xi_{t} \nabla\left(h_{t} \circ E\right)(y)=0, \quad\left(W_{E}\right) \\
\lambda \in R^{p}, \lambda \geqslant 0, \lambda e=1, e=(1,1, \cdots, 1)^{\top} \in R^{p}, \mu \in R^{m}, \mu \geqq 0, \xi \in R^{q},
\end{gathered}
$$

where all functions are defined in the similar way as for the considered vector optimization problem (VP) (3.1) and $e=(1, \cdots, 1) \in R^{p}$. Further, let

$$
\begin{aligned}
\Gamma_{E}=\left\{(y, \lambda, \mu, \xi) \in R^{n} \times R^{p} \times R^{m} \times R^{q}:\right. & \sum_{i=1}^{p} \lambda_{i} \nabla\left(f_{i} \circ E\right)(y)+\sum_{j=1}^{m} \mu_{j} \nabla\left(g_{j} \circ E\right)(y) \\
& \left.+\sum_{t=1}^{q} \xi_{t} \nabla\left(h_{t} \circ E\right)(y)=0, \lambda \geqslant 0, \lambda e=1, \mu \geqq 0\right\}
\end{aligned}
$$

be the set of all feasible solutions of the problem $\left(\mathrm{WD}_{E}\right)$ (4.1). Further, $Y_{E}=\left\{y \in X:(y, \lambda, \mu, \xi) \in \Gamma_{E}\right\}$. We call the vector dual problem $\left(\mathrm{WD}_{\mathrm{E}}\right)$ (4.1) Wolfe vector $\mathrm{E}$-dual problem or vector $\mathrm{E}$-dual problem in the sense of Wolfe.

Now, under E-convexity hypotheses, we prove duality results between the E-vector problems (VP) (3.1) and $\left(\mathrm{WD}_{\mathrm{E}}\right)$ (4.1) and, thus, E-duality results between the problems (VP) (3.1) and (WDE) (4.1).

Theorem 4.1 (Weak duality between $\left(\mathrm{VP}_{\mathrm{E}}\right)$ (3.2) and $\left(\mathrm{WD}_{\mathrm{E}}\right)$ (4.1) and also weak E-duality between (VP) (3.1) and $\left.\left(\mathrm{WD}_{\mathrm{E}}\right)(4.1)\right)$.

Let $z$ and $(y, \lambda, \mu, \xi)$ be any feasible solutions of the problems $\left(V P_{\mathrm{E}}\right)(3.2)$ and $\left(W D_{\mathrm{E}}\right)(4.1)$, respectively. Assume, moreover, that each objective function $f_{i}, i \in I$, is E-convex at $y$ on $\Omega_{E} \cup Y_{E}$, each constraint function $g_{j}, j \in J$, is E-convex at $y$ on $\Omega_{\mathrm{E}} \cup \mathrm{Y}_{\mathrm{E}}$, the functions $\mathrm{h}_{\mathrm{t}}, \mathrm{t} \in \mathrm{T}^{+}(\mathrm{E}(\mathrm{y}))$ and the functions $-\mathrm{h}_{\mathrm{t}}, \mathrm{t} \in \mathrm{T}^{-}(\mathrm{E}(\mathrm{y}))$, are $\mathrm{E}$-convex at y on $\Omega_{\mathrm{E}} \cup \mathrm{Y}_{\mathrm{E}}$. Then

$$
(f \circ E)(z) \nless \psi_{E}(y, \mu, \xi) .
$$

In other words, E-weak duality holds between the problems (VP) (3.1) and (WD $)(4.1)$, that is, for any feasible solutions $x$ and $(y, \lambda, \mu, \xi)$ of the problems $(V P)(3.1)$ and $\left(W D_{E}\right)(4.1)$, respectively, the following relation

$$
f(x) \nless \psi_{E}(y, \mu, \xi)
$$

is true.

Proof. Suppose, contrary to the result, that

$$
(f \circ E)(z)<\psi_{E}(y, \mu, \xi) .
$$

Thus,

$$
\left(f_{i} \circ E\right)(z)<\left(f_{i} \circ E\right)(y)+\left[\sum_{j=1}^{m} \mu_{j}\left(g_{j} \circ E\right)(y)+\sum_{t=1}^{q} \xi_{t}\left(h_{t} \circ E\right)(y)\right], i \in I .
$$

Multiplying by $\lambda_{i}$ and then adding both sides of the above inequalities and taking that $\sum_{i=1}^{p} \lambda_{i}=1$, we get the inequality

$$
\sum_{i=1}^{p} \lambda_{i}\left(f_{i} \circ E\right)(z)<\sum_{i=1}^{p} \lambda_{i}\left(f_{i} \circ E\right)(y)+\sum_{j=1}^{m} \mu_{j}\left(g_{j} \circ E\right)(y)+\sum_{t=1}^{q} \xi_{t}\left(h_{t} \circ E\right)(y)
$$


holds. From the feasibility of $z$ for the problem $\left(\mathrm{VP}_{\mathrm{E}}\right)(3.2)$, it follows that

$$
\begin{aligned}
& \sum_{i=1}^{p} \lambda_{i}\left(f_{i} \circ E\right)(z)+\sum_{j=1}^{m} \mu_{j}\left(g_{j} \circ E\right)(z)+\sum_{t=1}^{q} \xi_{t}\left(h_{t} \circ E\right)(z)< \\
& \sum_{i=1}^{p} \lambda_{i}\left(f_{i} \circ E\right)(y)+\sum_{j=1}^{m} \mu_{j}\left(g_{j} \circ E\right)(y)+\sum_{t=1}^{q} \xi_{t}\left(h_{t} \circ E\right)(y) .
\end{aligned}
$$

By assumption, $z$ and $(y, \lambda, \mu, \xi)$ are feasible solutions for the problems ( $\left.\mathrm{VP}_{\mathrm{E}}\right)$ (3.2) and $\left(\mathrm{WD}_{\mathrm{E}}\right)$ (4.1), respectively. Since the functions $f_{i}, i \in I, g_{j}, j \in J, h_{t}, t \in T^{+},-h_{t}, t \in T^{-}$, are E-convex on $\Omega_{E} \cup Y_{E}$, by Proposition 2.7, the following inequalities

$$
\begin{gathered}
\left(f_{i} \circ E\right)(z)-\left(f_{i} \circ E\right)(y) \geqq \nabla\left(f_{i} \circ E\right)(y)(E(z)-E(y)), i \in I, \\
\left(g_{j} \circ E\right)(z)-\left(g_{j} \circ E\right)(y) \geqq \nabla\left(g_{j} \circ E\right)(y)(E(z)-E(y)), j \in J_{E}(y), \\
\left(h_{t} \circ E\right)(z)-\left(h_{t} \circ E\right)(y) \geqq \nabla\left(h_{t} \circ E\right)(y)(E(z)-E(y)), t \in T^{+}(E(y)), \\
-\left(h_{t} \circ E\right)(z)+\left(h_{t} \circ E\right)(y) \geqq-\nabla\left(h_{t} \circ E\right)(y)(E(z)-E(y)), t \in T^{-}(E(y))
\end{gathered}
$$

hold, respectively. Multiplying inequalities (4.4), (4.5), (4.6), (4.7) by the corresponding Lagrange multipliers and then adding both sides of the resulting inequalities, we obtain that the inequality

$$
\begin{aligned}
& \sum_{i=1}^{p} \lambda_{i}\left(f_{i} \circ E\right)(z)-\sum_{i=1}^{p} \lambda_{i}\left(f_{i} \circ E\right)(y)+\sum_{j=1}^{m} \mu_{j}\left(g_{j} \circ E\right)(z) \\
& -\sum_{j=1}^{m} \mu_{j}\left(g_{j} \circ E\right)(y)+\sum_{t=1}^{q} \xi_{t}\left(h_{t} \circ E\right)(z)-\sum_{t=1}^{q} \xi_{t}\left(h_{t} \circ E\right)(y) \\
& \geqq\left[\sum_{i=1}^{p} \lambda_{i} \nabla\left(f_{i} \circ E\right)(y)+\sum_{j=1}^{m} \mu_{j} \nabla\left(g_{j} \circ E\right)(y)+\sum_{t=1}^{q} \xi_{t} \nabla\left(h_{t} \circ E\right)(y)\right](E(z)-E(y)),
\end{aligned}
$$

holds. Thus, by (4.3), it follows that the following inequality

$$
\left[\sum_{i=1}^{p} \lambda_{i} \nabla\left(f_{i} \circ E\right)(y)+\sum_{j=1}^{m} \mu_{j} \nabla\left(g_{j} \circ E\right)(y)+\sum_{t=1}^{q} \xi_{t} \nabla\left(h_{t} \circ E\right)(y)\right](E(z)-E(y))<0
$$

holds, contradicting the first constraint of the Wolfe vector $E$-dual problem $\left(W D_{E}\right)(4.1)$. This means that the proof of weak duality theorem between the E-vector optimization problems (VPE) (3.2) and (WDE (4.1) is completed. Then, the weak E-duality theorem between the problems (VP) (3.1) and (WDE) (4.1), that is, the relation (4.2) follows directly from Lemma 3.3. Thus, the proof of this theorem is completed.

If stronger E-convexity hypotheses are imposed on the functions constituting the considered vector optimization problems, then the stronger weak duality result is true.

Theorem 4.2 (Weak duality between $\left(\mathrm{VP}_{\mathrm{E}}\right)$ (3.2) and $\left(\mathrm{WD}_{\mathrm{E}}\right)$ (4.1) and also weak E-duality between (VP) (3.1) and $\left.\left(\mathrm{WD}_{\mathrm{E}}\right)(4.1)\right)$.

Let $z$ and $(y, \lambda, \mu, \xi)$ be any feasible solutions of the problems $\left(V P_{\mathrm{E}}\right)$ (3.2) and $\left(W D_{\mathrm{E}}\right)(4.1)$, respectively. Assume, moreover, that each objective function $f_{i}, i \in I$, is strictly $E$-convex at y on $\Omega_{E} \cup Y_{E}$, each constraint function $g_{j}$, $j \in J$, is $E$-convex at $y$ on $\Omega_{\mathrm{E}} \cup Y_{\mathrm{E}}$, the functions $h_{\mathrm{t}}, \mathrm{t} \in \mathrm{T}^{+}(\mathrm{E}(\mathrm{y}))$ and the functions $-\mathrm{h}_{\mathrm{t}}, \mathrm{t} \in \mathrm{T}^{-}(\mathrm{E}(\mathrm{y}))$, are $\mathrm{E}$-convex at $\mathrm{y}$ on $\Omega_{\mathrm{E}} \cup \mathrm{Y}_{\mathrm{E}}$. Then

$$
(f \circ E)(z) \nless \psi_{E}(y, \mu, \xi) .
$$

In other words, E-weak duality holds between the problems (VP) (3.1) and (WD $D_{\mathrm{E}}$ ) (4.1), that is, for any feasible solutions $x$ and $(y, \lambda, \mu, \xi)$ of the problems $(V P)$ (3.1) and (WDE (4.1), respectively,

$$
f(x) \nless \psi_{E}(y, \mu, \xi) \text {. }
$$


Remark 4.3. As it follows from the proofs of Theorems 4.1 and 4.2, the assumption of E-convexity of constraint functions can be weakened. Indeed, these results can be established if each constraint functions $g_{j}, j \in J, h_{t}, t \in T^{+}(y)$ and the functions $-h_{t}, t \in T^{-}(y)$, can be assumed to be quasi E-convex at $y$ on $\Omega_{E} \cup Y_{E}$.

Theorem 4.4 (Strong duality between $\left(\mathrm{VP}_{\mathrm{E}}\right)$ (3.2) and $\left(\mathrm{WD}_{\mathrm{E}}\right)(4.1)$ and also strong E-duality between (VP) (3.1) and $\left.\left(\mathrm{WD}_{\mathrm{E}}\right)(4.1)\right)$.

Let $\bar{x} \in \Omega_{\mathrm{E}}$ be a (weak) Pareto solution of the E-vector optimization problem (VP) (3.1) and the $\mathrm{E}$-Abadie constraint qualification (E-ACQ) be satisfied at $\bar{x}$. Then there exist $\bar{\lambda} \in \mathrm{R}^{\mathrm{p}}, \bar{\mu} \in \mathrm{R}^{\mathrm{m}}, \bar{\xi} \in \mathrm{R}^{\mathrm{q}}$ such that $(\overline{\mathrm{x}}, \bar{\lambda}, \bar{\mu}, \bar{\xi})$ is feasible for the problem $\left(W D_{\mathrm{E}}\right)(4.1)$ and the objective functions of $\left(V P_{\mathrm{E}}\right)(3.2)$ and $\left(W D_{\mathrm{E}}\right)(4.1)$ are equal at these points. If also all hypotheses of the weak duality theorem (Theorem 4.1 or Theorem 4.2) are satisfied, then $(\bar{x}, \bar{\lambda}, \bar{\mu}, \bar{\xi})$ is a (weak) efficient solution of maximum type for the problem ( $\left.W D_{\mathrm{E}}\right)(4.1)$.

In other words, in such a case, $\mathrm{E}(\overline{\mathrm{x}}) \in \Omega$ is a (weak) E-Pareto solution of the multiobjective programming problem $(V P)$ (3.1) and the strong E-duality holds between the problems (VP) (3.1) and (WDE (4.1).

Proof. By assumption, $\bar{x} \in \Omega_{\mathrm{E}}$ is a weak Pareto solution for the problem (VP $\mathrm{E}_{\mathrm{E}}$ ) (3.2) and the E-Abadie constraint qualification (E-ACQ) is satisfied at $\bar{x}$. Then, there exist Lagrange multiplier $\bar{\lambda} \in R^{p}, \bar{\mu} \in R^{m}$, $\bar{\xi} \in \mathrm{R}^{\mathrm{q}}$ such that the E-Karush-Kuhn-Tucker necessary optimality conditions (3.16), (3.17), (3.18) are satisfied at $\bar{x}$. Thus, the feasibility of $(\bar{x}, \bar{\lambda}, \bar{\mu}, \bar{\xi})$ in the problem $\left(\mathrm{WD}_{\mathrm{E}}\right)$ (4.1) follows directly from these conditions. Therefore, the objective functions for the problems (VP $\left.\mathrm{V}_{\mathrm{E}}\right)(3.2)$ and $\left(\mathrm{WD}_{\mathrm{E}}\right)(4.1)$ are equal at $\bar{x}$ and $(\bar{x}, \bar{\lambda}, \bar{\mu}, \bar{\xi})$, respectively. By the weak duality theorem (Theorem 4.1 or Theorem 4.2), it follows that the inequality $(f \circ E)(\bar{x}) \nless \psi_{E}(y, \mu, \xi)$ (or $\left.(f \circ E)(\bar{x}) \nless \psi_{E}(y, \mu, \xi)\right)$ is satisfied for any feasible point $(y, \lambda, \mu, \xi)$ of Wolfe vector E-dual problem $\left(\mathrm{WD}_{\mathrm{E}}\right)$ (4.1). Using the E-Karush-Kuhn-Tucker necessary optimality conditions (3.17) and (3.18) we get, for any feasible point $(y, \lambda, \mu, \xi)$ of the problem $\left(\mathrm{WD}_{\mathrm{E}}\right)(4.1)$, that

$$
\begin{aligned}
& (f \circ E)(\bar{x})+\left[\sum_{j=1}^{m} \mu_{j}\left(g_{j} \circ E\right)(\bar{x})+\sum_{t=1}^{q} \xi_{t}\left(h_{t} \circ E\right)(\bar{x})\right] e \\
& \quad \nless(f \circ E)(y)+\left[\sum_{j=1}^{m} \mu_{j}\left(g_{j} \circ E\right)(y)+\sum_{t=1}^{q} \xi_{t}\left(h_{t} \circ E\right)(y)\right] e .
\end{aligned}
$$

Hence, by (4.8), it follows that $(\bar{\chi}, \bar{\lambda}, \bar{\mu}, \bar{\xi})$ is a weak efficient point of maximum type for Wolfe vector E-dual problem $\left(\mathrm{WD}_{\mathrm{E}}\right)$ (4.1). The strong E-duality holds between the problems (VP) (3.1) and $\left(\mathrm{WD}_{\mathrm{E}}\right)$ (4.1) follows directly from Lemma 3.4. Namely, $E(\bar{x})$ is a weak E-Pareto (E-Pareto) solution of the vector optimization problem (VP) (3.1) and then $(\bar{x}, \bar{\lambda}, \bar{\mu}, \bar{\xi})$ is a (weak) efficient solution of maximum type for the problem $\left(\mathrm{WD}_{\mathrm{E}}\right)(4.1)$.

Theorem 4.5 (Converse duality between $\left(\mathrm{VP}_{\mathrm{E}}\right)$ (3.2) and $\left(\mathrm{WD}_{\mathrm{E}}\right)$ (4.1) and also converse E-duality between (VP) (3.1) and (WDE (4.1)).

Let $(\bar{x}, \bar{\lambda}, \bar{\mu}, \bar{\xi})$ be a (weak) efficient solution of a maximum type in the vector $\mathrm{E}-$ Wolfe dual problem $\left(W D_{\mathrm{E}}\right)(4.1)$ such that $\bar{x} \in \Omega_{\mathrm{E}}$. Moreover, that the objective functions $\mathrm{f}_{i}, i \in \mathrm{I}$, are (strictly) $\mathrm{E}$-convex at $\bar{x}$ on $\Omega_{\mathrm{E}} \cup \mathrm{Y}_{\mathrm{E}}$, the constraint functions $g_{j}, j \in J$, are $E$-convex at $\bar{x}$ on $\Omega_{E} \cup Y_{E}$, the functions $h_{t}, t \in T^{+}(E(\bar{x}))$ and the functions 
$-h_{\mathrm{t}}, \mathrm{t} \in \mathrm{T}^{-}(\mathrm{E}(\overline{\mathrm{x}}))$, are $\mathrm{E}-$ convex at $\overline{\mathrm{x}}$ on $\Omega_{\mathrm{E}} \cup \mathrm{Y}_{\mathrm{E}}$. Then $\overline{\mathrm{x}}$ is a weak Pareto (Pareto) solution of the problem $\left(V P_{\mathrm{E}}\right)$ (3.2) and, thus, $\mathrm{E}(\overline{\mathrm{x}})$ is a weak $\mathrm{E}$-Pareto (E-Pareto) solution of the problem (VP) (3.1).

Proof. Proof of this theorem follows directly from Theorem 4.1 (or Theorem 4.2).

Theorem 4.6 (Restricted converse duality between $\left(\mathrm{VP}_{\mathrm{E}}\right)(3.2)$ and $\left(\mathrm{WD}_{\mathrm{E}}\right)(4.1)$ and also restricted converse E-duality between (VP) (3.1) and (WDE) (4.1)).

Let $\bar{x}$ and $(\bar{y}, \bar{\lambda}, \bar{\mu}, \bar{\xi})$ be feasible solutions for the problems $\left(V P_{\mathrm{E}}\right)(3.2)$ and $\left(W D_{\mathrm{E}}\right)(4.1)$, respectively, such that

$$
(f \circ E)(\bar{x})<(f \circ E)(\bar{y})+\left[\sum_{j=1}^{m} \bar{\mu}_{j}\left(g_{j} \circ E\right)(\bar{y})+\sum_{t=1}^{q} \bar{\xi}_{t}\left(h_{t} \circ E\right)(\bar{y})\right] e . \quad(\leqslant)
$$

Moreover, assume that the objective functions $f_{i}, i \in I$, are (strictly) $E$-convex at $\bar{y}$ on $\Omega_{\mathrm{E}} \cup \mathrm{Y}_{\mathrm{E}}$, the constraint functions $g_{j}, j \in J$, are $E$-convex at $\bar{y}$ on $\Omega_{E} \cup Y_{E}$, the functions $h_{t}, t \in T^{+}(E(\bar{y}))$ and functions $-h_{t}, t \in$ $\mathrm{T}^{-}\left(\mathrm{E}(\overline{\mathrm{y}})\right.$ ), are $\mathrm{E}-$ convex at $\overline{\mathrm{y}}$ on $\Omega_{\mathrm{E}} \cup \mathrm{Y}_{\mathrm{E}}$. Then $\overline{\mathrm{x}}=\overline{\mathrm{y}}$, that is, $\overline{\mathrm{x}}$ is a weak Pareto (Pareto) solution of the problem $\left(V P_{\mathrm{E}}\right)(3.2)$ and $(\overline{\mathrm{y}}, \bar{\lambda}, \bar{\mu}, \bar{\xi})$ is a weak efficient point (efficient) of maximum type for the problem (WD $\left.\mathrm{E}\right)(4.1)$. In other words, $\mathrm{E}(\overline{\mathrm{x}})$ is a weak E-Pareto (E-Pareto) solution of the problem (VP) (3.1) and $(\overline{\mathrm{y}}, \bar{\lambda}, \bar{\mu}, \bar{\xi})$ is a weakly efficient (an efficient) solution of maximum type for the problem $\left(W D_{\mathrm{E}}\right)(4.1)$.

Proof. From (4.9), it follows that

$$
\left(f_{i} \circ E\right)(\bar{x})<\left(f_{i} \circ E\right)(\bar{y})+\sum_{j=1}^{m} \bar{\mu}_{j}\left(g_{j} \circ E\right)(\bar{y})+\sum_{t=1}^{q} \bar{\xi}_{t}\left(h_{t} \circ E\right)(\bar{y}), i \in I .
$$

Multiplying each inequality (4.10) by $\bar{\lambda}_{i}, i \in I$, and then adding both sides of the resulting inequalities, we get

$$
\sum_{i=1}^{p} \bar{\lambda}_{i}(f \circ E)(\bar{x})<\sum_{i=1}^{p} \bar{\lambda}_{i}(f \circ E)(\bar{y})+\left[\sum_{j=1}^{m} \bar{\mu}_{j}\left(g_{j} \circ E\right)(\bar{y})+\sum_{t=1}^{q} \bar{\xi}_{t}\left(h_{t} \circ E\right)(\bar{y})\right] \sum_{i=1}^{p} \lambda_{i} .
$$

Since $\sum_{i=1}^{p} \lambda_{i}=1,(4.11)$ implies

$$
\sum_{i=1}^{p} \bar{\lambda}_{i}\left(f_{i} \circ E\right)(\bar{x})<\sum_{i=1}^{p} \bar{\lambda}_{i}\left(f_{i} \circ E\right)(\bar{y})+\sum_{j=1}^{m} \bar{\mu}_{j}\left(g_{j} \circ E\right)(\bar{y})+\sum_{t=1}^{q} \bar{\xi}_{t}\left(h_{t} \circ E\right)(\bar{y}) .
$$

Now, we proceed by contradiction. Suppose, contrary to the result, that $\bar{x} \neq \bar{y}$. By assumption, the objective functions $f_{i}, i \in I(y)$, are E-convex at $\bar{y}$ on $\Omega_{E} \cup Y_{E}$, the constraint functions $g_{j}, j \in J(y)$, are E-convex at $\bar{y}$ on $\Omega_{E} \cup Y_{E}$, the functions $h_{t}, t \in T^{+}(E(\bar{y}))$ and functions $-h_{t}, t \in T^{-}(E(\bar{y}))$, are E-convex at $\bar{y}$ on $\Omega_{E} \cup Y_{E}$. Then, by Proposition 2.7, the following inequalities

$$
\begin{gathered}
\left(f_{i} \circ E\right)(\bar{x})-\left(f_{i} \circ E\right)(\bar{y}) \geqq \nabla\left(f_{i} \circ E\right)(\bar{y})(E(\bar{x})-E(\bar{y})), i \in I, \\
\left(g_{j} \circ E\right)(\bar{x})-\left(g_{j} \circ E\right)(\bar{y}) \geqq \nabla\left(g_{j} \circ E\right)(\bar{y})(E(\bar{x})-E(\bar{y})), j \in J(E(\bar{y})), \\
\left(h_{t} \circ E\right)(\bar{x})-\left(h_{t} \circ E\right)(\bar{y}) \geqq \nabla\left(h_{t} \circ E\right)(\bar{y})(E(\bar{x})-E(\bar{y})), t \in T^{+}(E(\bar{y})), \\
-\left(h_{t} \circ E\right)(\bar{x})+\left(h_{t} \circ E\right)(\bar{y}) \geqq-\nabla\left(h_{t} \circ E\right)(\bar{y})(E(\bar{x})-E(\bar{y})), t \in T^{-}(E(\bar{y}))
\end{gathered}
$$

hold, respectively. Multiplying inequalities (4.13), (4.14), (4.15), (4.16) by the corresponding Lagrange multipliers and then adding both sides of the resulting inequalities, we get

$$
\begin{aligned}
& \sum_{i=1}^{p} \bar{\lambda}_{i}\left(f_{i} \circ E\right)(\bar{x})-\sum_{i=1}^{p} \bar{\lambda}_{i}\left(f_{i} \circ E\right)(\bar{y})+\sum_{j=1}^{m} \bar{\mu}_{j}\left(g_{j} \circ E\right)(\bar{x}) \\
& \quad-\sum_{j=1}^{m} \bar{\mu}_{j}\left(g_{j} \circ E\right)(\bar{y})+\sum_{t=1}^{q} \bar{\xi}_{t}\left(h_{t} \circ E\right)(\bar{x})-\sum_{t=1}^{q} \bar{\xi}_{t}\left(h_{t} \circ E\right)(\bar{y}) \\
& \geqq\left[\sum_{i=1}^{p} \bar{\lambda}_{i} \nabla\left(f_{i} \circ E\right)(\bar{y})+\sum_{j=1}^{m} \bar{\mu}_{j} \nabla\left(g_{j} \circ E\right)(\bar{y})+\sum_{t=1}^{q} \bar{\xi}_{t} \nabla\left(h_{t} \circ E\right)(\bar{y})\right](E(\bar{x})-E(\bar{y})) .
\end{aligned}
$$


By (4.17) and the first constraint of $\left(\mathrm{WD}_{\mathrm{E}}\right)(4.1)$, it follows that

$$
\begin{aligned}
& \sum_{i=1}^{p} \bar{\lambda}_{i}\left(f_{i} \circ E\right)(\bar{x})+\sum_{j=1}^{m} \bar{\mu}_{j}\left(g_{j} \circ E\right)(\bar{x})+\sum_{t=1}^{q} \bar{\xi}_{t}\left(h_{t} \circ E\right)(\bar{x}) \\
& \quad \geqq \sum_{i=1}^{p} \bar{\lambda}_{i}\left(f_{i} \circ E\right)(\bar{y})+\sum_{j=1}^{m} \bar{\mu}_{j}\left(g_{j} \circ E\right)(\bar{y})+\sum_{t=1}^{q} \bar{\xi}_{t}\left(h_{t} \circ E\right)(\bar{y}) .
\end{aligned}
$$

Hence, by $\bar{x} \in \Omega_{\mathrm{E}}$, we get that the following inequality

$$
\sum_{i=1}^{p} \bar{\lambda}_{i}\left(f_{i} \circ E\right)(\bar{x}) \geqq \sum_{i=1}^{p} \bar{\lambda}_{i}\left(f_{i} \circ E\right)(\bar{y})+\sum_{j=1}^{m} \bar{\mu}_{j}\left(g_{j} \circ E\right)(\bar{y})+\sum_{t=1}^{q} \bar{\xi}_{t}\left(h_{t} \circ E\right)(\bar{y})
$$

holds, contradicting (4.12). Then, $\bar{x}=\bar{y}$ and this means by weak duality (Theorem 4.1) that $\bar{x}$ is a weak Pareto solution of the problem $\left(\mathrm{VP}_{\mathrm{E}}\right)(3.2)$ and $(\bar{y}, \bar{\lambda}, \bar{\mu}, \bar{\xi})$ is a weak efficient solution of maximum type for the problem $\left(W D_{E}\right)$ (4.1). Further, by Lemma 3.4, it follows that $E(\bar{x})$ is a weak E-Pareto solution of the problem $\left(\mathrm{VP}_{\mathrm{E}}\right)(3.2)$ and $(\bar{y}, \bar{\lambda}, \bar{\mu}, \bar{\xi})$ is a weak efficient solution of maximum type for the problem $\left(\mathrm{WD}_{E}\right)$ (4.1). Thus, the proof of this theorem is completed.

\section{Concluding remarks}

In this paper, a nonlinear multiobjective programming problem with both inequality and equality constraints has been considered in which the involved functions are not necessarily differentiable, but they are E-differentiable. The so-called E-Fritz John necessary optimality conditions have been established for the considered E-differentiable vector optimization problem with both inequality and equality constraints. Further, under the introduced E-Abadie constraint qualification, also the so-called E-Karush-Kuhn-Tucker necessary optimality conditions have been established for such a not necessarily differentiable multiobjective programming problem. In order to illustrate this result an example of such a vector optimization problem with E-differentiable functions has been given in which the E-Abadie constraint qualification is not satisfied and, therefore, the E-Karush-Kuhn-Tucker necessary optimality conditions are not satisfied in such a case. Also several sufficient optimality conditions have been derived for such nonlinear vector optimization problems with E-differentiable functions under (generalized) E-convexity hypotheses.

Further, the so-called Wolfe E-duality theory has been investigated for the considered E-differentiable vector optimization problem with both inequality and equality constraints. Various E-duality theorems between the E-differentiable vector optimization problem and its vector Wolfe E-dual problem have been proved also under (generalized) E-convexity hypotheses.

However, some interesting topics for further research remain. It would be of interest to investigate whether it is possible to prove similar optimality results for other classes of E-differentiable vector optimization problems. We shall investigate these questions in subsequent papers.

\section{References}

[1] B. Aghezzaf, M. Hachimi, Sufficient optimality conditions and duality in multiobjective optimization involving generalized convexity, Numer. Funct. Anal. Optim., 22 (2006), 775-788. 1

[2] I. Ahmad, Sufficiency and duality in multiobjective programming with generalized (F, $\rho)$-convexity, J. Appl. Anal., 11 (2005), 19-33. 1

[3] T. Antczak, On G-invex multiobjective programming. I. Optimality, J. Global Optim., 43 (2009), 97-109. 1

[4] T. Antczak, On G-invex multiobjective programming. II. Duality, J. Global Optim., 43 (2009), 111-140. 1

[5] T. Antczak, On nonsmooth $(\Phi, \rho)$-invex multiobjective programming in finite-dimensional Euclidean spaces, J. Adv. Math. Stud., 7 (2014), 127-145. 1

[6] T. Antczak, Multiobjective programming under nondifferentiable G-V-invexity, Filomat, 30 (2016), 2909-2923. 1

[7] T. Antczak, Optimality conditions in quasidifferentiable vector optimization, J. Optim. Theory Appl., 171 (2016), 708 725. 1 
[8] T. Antczak, M. A. Jiménez, KT-G-invexity in multiobjective programming, Int. J. Math. Comput., 27 (2016), 23-39. 1

[9] M. Arana-Jiménez, Efficiency under pseudoinvexity and duality in differentiable and non-differentiable multiobjective problems, A characterization, in: Recent developments on mathematical programming and applications, 2009 (2009), $1-14.1$

[10] M. Arana-Jiménez, G. R. Garzón, A. R. Lizana, Optimality conditions in vector optimization, Bentham Science Publishers, Oak Park, (2010). 1

[11] X. S. Chen, Some properties of semi-E-convex functions, J. Math. Anal. Appl., 275 (2002), 251-262. 1

[12] T. D. Chuong, D. S. Kim, Optimality conditions and duality in nonsmooth multiobjective optimization problems, Ann. Oper. Res., 217 (2014), 117-136. 1

[13] G. Giorgi, A. Guerraggio, The notion of invexity in vector optimization: smooth and nonsmooth case, in: Generalized Convexity, Generalized Monotonicity: Recent Results, 1998 (1998), 389-405.

[14] N. J. Huang, J. Li, S. Y. Wu, Optimality conditions for vector optimization problems, J. Optim. Theory Appl., 142 (2009), 323-342. 1

[15] A. Iqbal, I. Ahmad, S. Ali, Some properties of geodesic semi-E-convex functions, Nonlinear Anal., 74 (2011), $6805-6813$.

[16] A. Iqbal, S. Ali, I. Ahmad, On geodesic E-convex sets, geodesic E-convex functions and E-epigraphs, J. Optim. Theory Appl., 155 (2012), 239-251.

[17] J. Jahn, Vector optimization: theory, applications and extensions, Springer-Verlag, Berlin, (2011). 1

[18] V. Jeyakumar, B. Mond, On generalised convex mathematical programming, J. Austral. Math. Soc. Ser. B, 34 (1992), 43-53. 1

[19] P. Kanniappan, Necessary conditions for optimality of nondifferentiable convex multiobjective programming, J. Optim. Theory Appl., 40 (1983), 167-174. 1

[20] R. N. Kaul, S. K. Suneja, M. K. Srivastava, Optimality criteria and duality in multiple-objective optimization involving generalized invexity, J. Optim. Theory Appl., 80 (1994), 465-482.

[21] P. Q. Khanh, T. H. Nuong, On necessary optimality conditions in vector optimization problems, J. Optim. Theory Appl., 58 (1988), 63-81. 1

[22] D. S. Kim, S. Schaible, Optimality and duality for invex nonsmooth multiobjective programming problems, Optimization, 53 (2004), 165-176. 1

[23] H. Kuk, G. M. Lee, D. S. Kim, Nonsmooth multiobjective programs with V-p-invexity, Indian J. Pure Appl. Math., 29 (1998), 405-412. 1

[24] G. M. Lee, Nonsmooth invexity in multiobjective programming, J. Inform. Optim. Sci., 15 (1994), 127-136. 1

[25] Z. Li, The optimality conditions of differentiable vector optimization problems, J. Math. Anal. Appl., 201 (1996), 35-43. 1

[26] J. G. Lin, Maximal vectors and multi-objective optimization, J. Optimization Theory Appl., 18 (1976), 41-64.

[27] D. T. Luc, Theory of vector optimization, Springer-Verlag, Berlin, (1989). 1

[28] O. L. Mangasarian, Nonlinear programming, Society for Industrial and Applied Mathematics (SIAM), Philadelphia, (1994). 3.7

[29] A. A. Megahed, A.-Z. El-Banna, E. A. Youness, H. G. Gomaa, A combined interactive approach for solving E-convex multiobjective nonlinear programming problem, Appl. Math. Comput., 217 (2011), 6777-6784. 1

[30] A. A. Megahed, H. G. Gomma, E. A. Youness, A. H. El-Banna, Optimality conditions of E-convex programming for an E-differentiable function, J. Inequal. Appl., 2013 (2013), 11 pages. 1, 2.6

[31] F. Mirzapour, Some properties on E-convex and E-quasi-convex functions, The 18th Seminar on Mathematical Analysis and its Applications (Moallem University), 2009 (2009), 178-181.

[32] S. K. Mishra, S.-Y. Wang, K. K. Lai, Generalized convexity and vector optimization, Springer-Verlag, Berlin, (2009). 1

[33] S. Nobakhtian, Infine functions and nonsmooth multiobjective optimization problems, Comput. Math. Appl., 51 (2006), 1385-1394. 1

[34] G.-R. Piao, L. G. Jiao, D. S. Kim, Optimality and mixed duality in multiobjective E-convex programming, J. Inequal. Appl., 2015 (2015), 13 pages. 1

[35] R. T. Rockafellar, The theory of subgradients and its applications to problems of optimization, Heldermann Verlag, Berlin, (1981). 3

[36] J. Sheiba Grace, P. Thangavelu, Properties of E-convex sets, Tamsui Oxf. J. Math. Sci., 25 (2009), 1-7.

[37] M. Soleimani-Damaneh, E-convexity and its generalizations, Int. J. Comput. Math., 88 (2011), 3335-3349. 1

[38] Y.-R. Syau, E. S. Lee, Some properties of E-convex functions, Appl. Math. Lett., 18 (2005), 1074-1080.

[39] X. M. Yang, On E-convex sets, E-convex functions, and E-convex programming, J. Optim. Theory Appl., 109 (2001), 699-704. 1

[40] E. A. Youness, E-convex sets, E-convex functions, and E-convex programming, J. Optim. Theory Appl., 102 (1999), 439-450. 1, 2, 2.1, 2.2

[41] E. A. Youness, Optimality criteria in E-convex programming, Chaos Solitons Fractals, 12 (2001), 1737-1745.

[42] E. A. Youness, Quasi and strictly quasi E-convex functions, J. Stat. Manag. Syst., 4 (2001), 201-210. 2, 2.4

[43] E. A. Youness, Characterization of efficient solutions of multi-objective E-convex programming problems, Appl. Math. Comput., 151 (2004), 755-761. 1

[44] E. A. Youness, T. Emam, Strongly E-convex sets and strongly E-convex functions, J. Interdiscip. Math., 8 (2005), 107-117. 1 